\title{
Multiple-Attribute Decision-Making Using Fermatean Fuzzy Hamacher Interactive Geometric Operators
}

\author{
Gulfam Shahzadi $\mathbb{D}^{1},{ }^{1}$ Fariha Zafar $\mathbb{D D}^{2}$ and Maha Abdullah Alghamdi $\mathbb{D}^{3}$ \\ ${ }^{1}$ Department of Mathematics, Virtual University of Pakistan, Lahore, Pakistan \\ ${ }^{2}$ Department of Mathematics, University of Okara, Okara, Pakistan \\ ${ }^{3}$ Department of Mathematics, Faculty of Science, Imam Abdulrahman Bin Faisal University, Dammam, Saudi Arabia
}

Correspondence should be addressed to Fariha Zafar; fariha.zafar@uo.edu.pk

Received 22 April 2021; Revised 31 May 2021; Accepted 8 June 2021; Published 25 June 2021

Academic Editor: Feng Feng

Copyright (c) 2021 Gulfam Shahzadi et al. This is an open access article distributed under the Creative Commons Attribution License, which permits unrestricted use, distribution, and reproduction in any medium, provided the original work is properly cited.

\begin{abstract}
Fermatean fuzzy set (FFS) is a more efficient, flexible, and generalized model to deal with uncertainty, as compared to intuitionistic and Pythagorean fuzzy models. This research article presents a novel multiple-attribute decision-making (MADM) technique based on FFS. Aggregation operators (AOs), for example, Dombi, Einstein, and Hamacher, are frequently being used in the MADM process and are considered useful tools for evaluating the given alternatives. Among these, one of the most effective is the Hamacher operator. The salient feature of this operator is that it reduces the impact of negative information and provides more accurate results. Motivated by the primary characteristics of the Hamacher operator, we apply Hamacher interactive aggregation operators based on FFSs to determine the best alternative. Using Hamacher's norm operations, we introduce some new geometric operators, namely, Fermatean fuzzy Hamacher interactive weighted geometric (FFHIWG) operator, Fermatean fuzzy Hamacher interactive ordered weighted geometric (FFHIOWG) operator, and Fermatean fuzzy Hamacher interactive hybrid weighted geometric (FFHIHWG) operator. Some important results and properties of the proposed AOs are discussed, and to achieve the optimal alternative, the proposed MADM technique is carried out in a real-life application of the medical field. An algorithm of the proposed technique is also developed. The significance of the proposed method is that Fermatean fuzzy Hamacher interactive geometric (FFHIG) operators deal with the relationship among belongingness degree (BD) and nonbelongingness degree (NBD) of the objects, which perform a crucial role in decision-making (DM). At last, to show the exactness and validity of the proposed work, a comparative analysis of the proposed model and the existing models is presented.
\end{abstract}

\section{Introduction}

Ambiguous or uncertain information is one of the greatest dilemmas dealing with the MADM process. The uncertain information can be captured in different ways. In the last few years, Zadeh's fuzzy set theory (FST) [1] and its extensions, i.e., intuitionistic fuzzy set theory (IFST) [2], Pythagorean fuzzy set theory (PFST) [3], hesitant fuzzy set theory (HFST) [4, 5], and intervalvalued fuzzy set theory (IVFST) [6], have been proved to be efficient tools handling uncertainty in numerous applications of MADM. However, there are some restrictions involved in all these theories, for example, FST deals with belongingness degree only, whereas IFST deals with both $\mathrm{BD}$ and NBD but it restricts their sum to be less or equal to 1 . To overcome this issue, PFST replaces the condition of the sum to "the sum of squares of $\mathrm{BD}$ and NBD to be less or equal to 1 ." Recently, a more generalized theory, namely, Fermatean fuzzy set theory (FFST), was introduced by Senapati and Yager [7]. The notion of FFS was initiated from IFSs and PFSs, where the sum of cubes of NBD and BD is less than or equal to one. Therefore, FFSs are more flexible and generalized as compared to both IFSs and PFSs.

Aggregation methods based on IFSs and PFSs were widely used in MADM. Xu [8] and Zhao et al. [9] defined aggregation operators (AOs) based on IFSs. Wei and Lu [10] developed Pythagorean fuzzy (PF) power AOs and used 
them in DM problems. Ordered weighted averaging aggregation operators (OWAAOs) for MADM were defined by Yager [11]. Many multiple-attribute decision-making models (MADMMs) use algebraic operators and these are Dombi, Einstein, and Hamacher operators. In recent years, many theories were developed based on these operators. Dombi [12] defined Dombi triangular norm and conorm operators. Many authors contributed their work to Dombi AOs. Akram et al. [13] worked on Pythagorean Dombi fuzzy AOs (PFDAOs). Wei [14] introduced interaction AOs based on PFSs and also applied these to MADMMs.

Hamacher AOs were introduced in 1978 [15]. Wei [16] defined Hamacher AOs based on PFSs and gave a comparative analysis for MADM. Garg $[17,18]$ presented some series of IF interactive averaging AOs by applying interactive averaging AOs on IFSs and also gave the idea of IF Hamacher AOs having entropy weight. Wu and Wei [19] presented MADMMs based on PF Hamacher AOs (PFHAOs). Wei [14] introduced the PF interaction AOs (PFIAOs) with their application to MADM. Waseem et al. [20] discussed MADM based on $m$-polar fuzzy Hamacher AOs (mFHAOs). Zhao and Wei [21] gave the idea of IF Einstein hybrid AOs (IFEHAOs). Wang and Liu [22] elaborated IF information AOs using Einstein operations. The idea for assessment of express service quality with entropy weight was explained by Wang et al. [23] under PF interactive Hamacher power AO.

Senapati and Yager [24] introduced Fermatean fuzzy averaging/geometric operators (FFAOS/FFFOs). They also defined operations over Fermatean fuzzy numbers (FFNs) [25]. Garg et al. [26] presented a method for the most suitable laboratory selection for COVID-19 test under Fermatean fuzzy environment (FFE). Akram et al. [27] discussed a MADMM to show the benefits of a sanitizer in COVID-19 under FFE. Shahzadi and Akram [28] proposed the idea of Fermatean fuzzy soft AOs and applied this idea in the field of group decision-making for the selection of an antivirus mask. Recently, Aydemir and Gunduz [29] explained the Fermatean fuzzy TOPSIS (FFTOPSIS) method consisting of Dombi AOs. Shahzadi et al. [30] introduced the idea of Hamacher interactive hybrid weighted averaging operators under FFE. Feng et al. [31] investigated membership grades of $q$-rung orthopair fuzzy sets geometrically. For more comprehension and understanding, the readers are referred to study [24, 25, 32-35].

The motivations of this study are defined as follows:

(i) The proposed Hamacher interactive AOs (HIAOs) deal with the relationship between the BD and NBD of an object

(ii) The MADMM based on FFSs shows that the change in NBDs will definitely affect the BDs of the objects

(iii) The proposed Fermatean fuzzy AOs generalize the BDs and NBDs of the objects; i.e., greater values of belongingness and nonbelongingness degrees can be taken as compared to IFST and PFST

(iv) The HIAOs are a much convenient approach to cope with the issues in the DM process; this article aims to define HIAOs based on FFSs to handle uncertainty associated with the choice of alternatives in MADMMs

(v) Hamacher interactive AOs give more precise and exact choice values in decision results when applied to MADMMs

The contributions of this article are outlined as follows:

(i) Some new HIAOs such as FFHIWG, FFHIOWG, and FFHIHWG are proposed here

(ii) The attractive properties alongside their special cases are discussed which reduce the loopholes in the existing operators

(iii) An algorithm for MADM using the proposed operators is described and an application is presented to show the applicability of the intended method in the real world

(iv) A comparison is also presented which shows the innovation and importance of the contemplated model

The remaining part of the article is arranged as follows. In Section 2, some elementary notions are presented. Section 3 explains a hybrid structure of Hamacher interactive operators based on FFSs such as FFHIWG operators with a few important results and basic properties, for example, boundedness, homogeneity, idempotency, monotonicity, and shift invariance. In Section 4, the basic concept and results of the FFHIOWG operator are presented. Section 5 presents the notions related to the FFHIHWG operator. In Section 6, a MADMM under Fermatean fuzzy environment is explained through a real-life application. In Section 7, the influence of distinct values of the parameter is shown. In Section 8, a comparative analysis with existing theories is discussed which shows the efficacy and importance of the intended model. In Section 9, the presented work is summarized with concluding remarks.

\section{Preliminaries}

Definition 1 (see [7]). A Fermatean fuzzy set (FFS) $\mathscr{L}$ on $\mathfrak{D}$ (a nonempty crisp set) is defined as

$$
\mathscr{L}=\left\{\left\langle\mathfrak{a}, \mu_{\mathscr{L}}(\mathfrak{a}), v_{\mathscr{L}}(\mathfrak{a})\right\rangle\right\}
$$

where $\quad \mu_{\mathscr{L}}: \mathfrak{D} \longrightarrow[0,1], \quad v_{\mathscr{L}}: \mathfrak{D} \longrightarrow[0,1] \quad$ and $\omega_{\mathscr{L}}(\mathfrak{a})=\sqrt[3]{1-\left(\mu_{\mathscr{L}}(\mathfrak{a})\right)^{3}-\left(\nu_{\mathscr{L}}(\mathfrak{a})\right)^{3}}$ indicate belongingness degree, nonbelongingness degree, and indeterminacy degree, respectively.

Definition 2 (see [7]). The score function (SF) and accuracy function $(\mathrm{AF})$ for a FFS $\mathscr{L}=\left(\mu_{\mathscr{L}}, v_{\mathscr{L}}\right)$ are given by

$$
\begin{array}{ll}
S(\mathscr{L})=\mu_{\mathscr{L}}^{3}-v_{\mathscr{L}}^{3}, & S(\mathscr{L}) \in[-1,1], \\
\mathscr{A}(\mathscr{L})=\mu_{\mathscr{L}}^{3}+v_{\mathscr{L}}^{3}, & \mathscr{A}(\mathscr{L}) \in[0,1] .
\end{array}
$$


Definition 3 (see [17]). Consider two FFSs $\mathscr{L}_{1}=\left\langle\mu_{\mathscr{L}_{1}}, v_{\mathscr{L}_{1}}\right\rangle$ and $\mathscr{L}_{2}=\left\langle\mu_{\mathscr{L}_{2}}, \nu_{\mathscr{L}_{2}}\right\rangle$. Then,

(1) If $S\left(\mathscr{L}_{1}\right)<S\left(\mathscr{L}_{2}\right)$, then $\mathscr{L}_{1} \prec \mathscr{L}_{2}$.

(2) If $S\left(\mathscr{L}_{1}\right)>S\left(\mathscr{L}_{2}\right)$, then $\mathscr{L}_{1}>\mathscr{L}_{2}$.

(3) If $S\left(\mathscr{L}_{1}\right)=S\left(\mathscr{L}_{2}\right)$, then

(a) If $\mathscr{A}\left(\mathscr{L}_{1}\right)<\mathscr{A}\left(\mathscr{L}_{2}\right)$, then $\mathscr{L}_{1} \prec \mathscr{L}_{2}$.

(b) If $\mathscr{A}\left(\mathscr{L}_{1}\right)>\mathscr{A}\left(\mathscr{L}_{2}\right)$, then $\mathscr{L}_{1}>\mathscr{L}_{2}$.

(c) If $\mathscr{A}\left(\mathscr{L}_{1}\right)=\mathscr{A}\left(\mathscr{L}_{2}\right)$, then $\mathscr{L}_{1} \sim \mathscr{L}_{2}$.

Definition 4 (see [15]). Hamacher t-norm and t-conorm are defined by

$$
\begin{aligned}
T\left(\mathfrak{a}_{\mathfrak{i}}, \mathfrak{a}_{\mathfrak{i}}\right) & =\frac{\mathfrak{a}_{\mathfrak{i}} \mathfrak{a}_{\mathfrak{i}}}{\varsigma+(1-\varsigma)\left(\mathfrak{a}_{\mathfrak{i}}+\mathfrak{a}_{\mathfrak{i}}-\mathfrak{a}_{\mathfrak{i}} \mathfrak{a}_{\mathfrak{i}}\right)}, \\
T^{*}\left(\mathfrak{a}_{\mathfrak{i}}, \mathfrak{a}_{j}\right) & =\frac{\mathfrak{a}_{\mathfrak{i}}+\mathfrak{a}_{\mathfrak{i}}-\mathfrak{a}_{\mathfrak{i}} \mathfrak{a}_{\mathfrak{i}}-(1-\varsigma) \mathfrak{a}_{\mathfrak{i}} \mathfrak{a}_{\mathfrak{j}}}{1-(1-\varsigma) \mathfrak{a}_{\mathfrak{i}} \mathfrak{a}_{\mathfrak{i}}} .
\end{aligned}
$$

(i) For $\varsigma=1$, these operations become algebraic t-norm, $T\left(\mathfrak{a}_{\mathfrak{i}}, \mathfrak{a}_{\mathfrak{j}}\right)=\mathfrak{a}_{\mathfrak{i}} \mathfrak{a}_{\mathfrak{i}}$, and algebraic $\mathrm{t}$-conorm, $T^{*}\left(\mathfrak{a}_{\mathfrak{i}}, \mathfrak{a}_{\mathfrak{j}}\right)=\mathfrak{a}_{\mathfrak{i}}+\mathfrak{a}_{\mathrm{i}}-\mathfrak{a}_{\mathfrak{i}} \mathfrak{a}_{\mathfrak{i}}$

(ii) For $\varsigma=2$, these operations become Einstein t-norm, $T\left(\mathfrak{a}_{\mathfrak{i}}, \mathfrak{a}_{\mathfrak{i}}\right)=\left(\mathfrak{a}_{\mathfrak{i}} \mathfrak{a}_{\mathfrak{i}} / 1+\left(1-\mathfrak{a}_{\mathfrak{i}}\right)\left(1-\mathfrak{a}_{\mathfrak{j}}\right)\right)$, and Einstein t-conorm, $T^{*}\left(\mathfrak{a}_{\mathfrak{i}}, \mathfrak{a}_{\mathfrak{j}}\right)=\left(\mathfrak{a}_{\mathfrak{i}}+\mathfrak{a}_{\mathfrak{i}} / 1+\mathfrak{a}_{\mathfrak{i}} \mathfrak{a}_{\mathfrak{i}}\right)$

Definition 5. Let $\mathscr{L}_{1}=\left\langle\mu_{1}, \nu_{1}\right\rangle, \quad \mathscr{L}_{2}=\left\langle\mu_{2}, v_{2}\right\rangle$, and $\mathscr{L}=\langle\mu, \nu\rangle$ be three FFSs and $\lambda>0$; then, (i) $\mathscr{L}_{1} \oplus \mathscr{L}_{2}=$

$\left\langle\sqrt[3]{\left(\prod_{i=1}^{2}\left(1+(\varsigma-1) \mu_{i}^{3}\right)-\prod_{i=1}^{2}\left(1-\mu_{i}^{3}\right) / \prod_{i=1}^{2}\left(1+(\varsigma-1) \mu_{i}^{3}\right)+(\varsigma-1) \prod_{i=1}^{2}\left(1-\mu_{i}^{3}\right)\right)}\right.$,

$\left.\sqrt[3]{\left(\varsigma\left\{\prod_{i=1}^{2}\left(1-\mu_{i}^{3}\right)-\prod_{i=1}^{2}\left(1-\mu_{i}^{3}-v_{i}^{3}\right)\right\} / \prod_{i=1}^{2}\left(1+(\varsigma-1) \mu_{i}^{3}\right)+(\varsigma-1) \prod_{i=1}^{2}\left(1-\mu_{i}^{3}\right)\right)}\right\rangle$

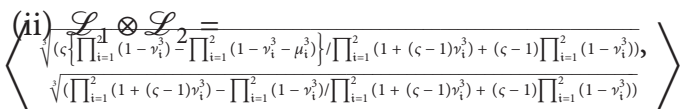

(iii) $\lambda \cdot \mathscr{L}=\left\langle\begin{array}{l}\sqrt[3]{\left(\left(1+(\varsigma-1) \mu^{3}\right)^{\lambda}-\left(1-\mu^{3}\right)^{\lambda} /\left(1+(\varsigma-1) \mu^{3}\right)^{\lambda}+(\varsigma-1)\left(1-\mu^{3}\right)^{\lambda}\right)}, \\ \sqrt[3]{\left(\varsigma\left\{\left(1-\mu^{3}\right)^{\lambda}-\left(1-\mu^{3}-\nu^{3}\right)^{\lambda}\right\} /\left(1+(\varsigma-1) \mu^{3}\right)^{\lambda}+(\varsigma-1)\left(1-\mu^{3}\right)^{\lambda}\right)}\end{array}\right\rangle$

(iv) $\mathscr{L}^{\lambda}=\left\langle\begin{array}{c}\sqrt[3]{\left(\left\{\left\{\left(1-\nu^{3}\right)^{\lambda}-\left(1-v^{3}-\mu^{3}\right)^{\lambda}\right\} /\left(1+(\varsigma-1) v^{3}\right)^{\lambda}+(\varsigma-1)\left(1-\nu^{3}\right)^{\lambda}\right)\right.}, \\ \sqrt[3]{\left(\left(1+(\varsigma-1) v^{3}\right)^{\lambda}-\left(1-v^{3}\right)^{\lambda} /\left(1+(\varsigma-1) v^{3}\right)^{\lambda}+(\varsigma-1)\left(1-\nu^{3}\right)^{\lambda}\right)}\end{array}\right\rangle$

\section{Fermatean Fuzzy Hamacher Interactive Weighted Geometric Operators}

In this section, we introduce the Fermatean fuzzy Hamacher interactive weighted geometric operator (FFHIWGO) and describe its some important characteristics.

Definition 6. Let $\mathscr{L}_{\mathfrak{i}}=\left(\mu_{\mathfrak{i}}, \nu_{\mathfrak{i}}\right)(\mathfrak{i}=1,2, \ldots, \mathfrak{y})$ be a family of FFSs and $\kappa=\left(\kappa_{1}, \kappa_{2}, \ldots, \kappa_{\mathfrak{y}}\right)^{T}$ be its weight vector (WV) such that $\kappa_{\mathfrak{i}}>0$ and $\sum_{\mathfrak{i}=1}^{\mathfrak{y}} \kappa_{\mathfrak{i}}=1$, then FFHIWG: $\Omega^{\mathfrak{y}} \longrightarrow \Omega$ is defined as

$$
\operatorname{FFHIWG}\left(\mathscr{L}_{1}, \mathscr{L}_{2}, \ldots, \mathscr{L}_{\mathfrak{y}}\right)=\kappa_{1} \mathscr{L}_{1} \otimes \kappa_{2} \mathscr{L}_{2} \otimes \cdots \otimes \kappa_{\mathfrak{y}} \mathscr{L}_{\mathfrak{y}} .
$$

Theorem 1. Let $\mathscr{L}_{\mathfrak{i}}=\left(\mu_{\mathfrak{i}}, \nu_{\mathfrak{i}}\right)$ be a collection of FFSs; then,

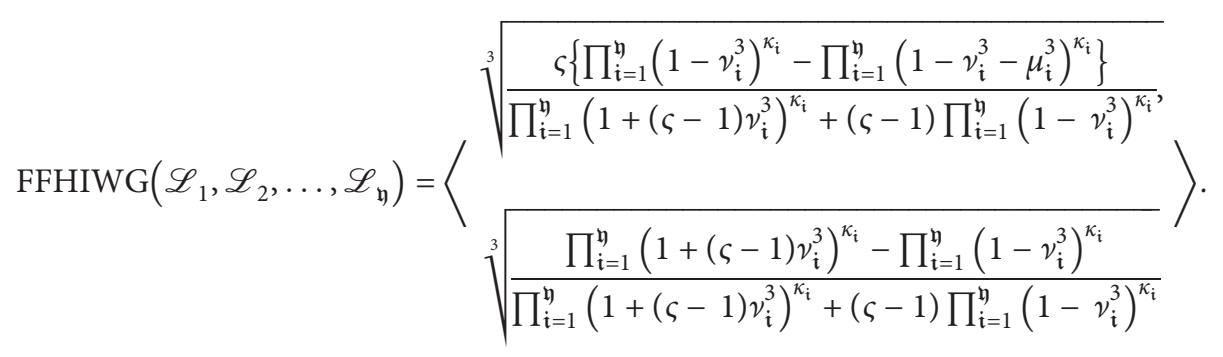

Proof. For $\mathfrak{y}=1, \kappa=\kappa_{1}=1$,

$$
\begin{aligned}
\operatorname{FFHIWG}\left(\mathscr{L}_{1}\right) & =\kappa_{1} \mathscr{L}_{1} \\
& =\mathscr{L}_{1} \\
& =\left(\mu_{1}, v_{1}\right) \\
& =\left\langle\sqrt[3]{\frac{\varsigma\left\{\left(1-v_{1}^{3}\right)-\left(1-v_{1}^{3}-\mu_{1}^{3}\right)\right\}}{\left(1+(\varsigma-1) v_{1}^{3}\right)+(\varsigma-1)\left(1-v_{1}^{3}\right)}}, \sqrt[3]{\left.\frac{\left(1+(\varsigma-1) v_{1}^{3}\right)-\left(1-v_{1}^{3}\right)}{\left(1+(\varsigma-1) \nu_{1}^{3}\right)+(\varsigma-1)\left(1-v_{1}^{3}\right)}\right\rangle} .\right.
\end{aligned}
$$


Thus, the result is true for $\mathfrak{y}=1$. Suppose that result holds for $\mathfrak{y}=\mathfrak{p}$, i.e.,

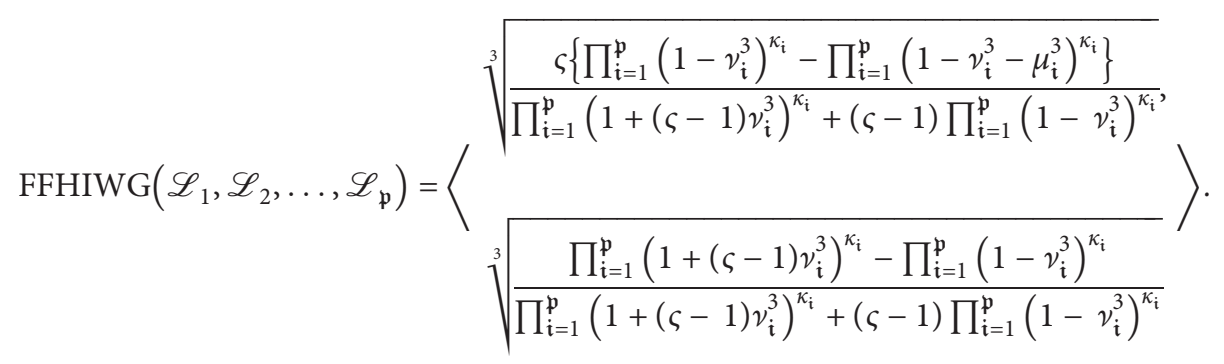

Now, for $\mathfrak{y}=\mathfrak{p}+1$,

$$
\begin{aligned}
& \operatorname{FFHIWG}\left(\mathscr{L}_{1}, \mathscr{L}_{2}, \ldots, \mathscr{L}_{\mathfrak{p}+1}\right)=\oplus_{\mathfrak{i}=1}^{\mathfrak{p}+1} \kappa_{\mathfrak{i}} \mathscr{L}_{\mathfrak{i}} \\
& =\left\langle\sqrt[3]{\frac{\varsigma\left\{\prod_{\mathfrak{i}=1}^{\mathfrak{p}}\left(1-\nu_{\mathfrak{i}}^{3}\right)^{\kappa_{\mathfrak{i}}}-\prod_{\mathfrak{i}=1}^{\mathfrak{p}}\left(1-v_{\mathfrak{i}}^{3}-\mu_{\mathfrak{i}}^{3}\right)^{\kappa_{\mathfrak{i}}}\right\}}{\prod_{\mathfrak{i}=1}^{\mathfrak{p}}\left(1+(\varsigma-1) \nu_{\mathfrak{i}}^{3}\right)^{\kappa_{\mathfrak{i}}}+(\varsigma-1) \prod_{\mathfrak{i}=1}^{\mathfrak{p}}\left(1-v_{\mathfrak{i}}^{3}\right)^{\kappa_{\mathfrak{i}}}},}\right\rangle \\
& \sqrt[3]{\frac{\prod_{\mathfrak{i}=1}^{\mathfrak{p}}\left(1+(\varsigma-1) v_{\mathfrak{i}}^{3}\right)^{\kappa_{\mathfrak{i}}}-\prod_{\mathfrak{i}=1}^{\mathfrak{p}}\left(1-v_{\mathfrak{i}}^{3}\right)^{\kappa_{\mathfrak{i}}}}{\prod_{\mathfrak{i}=1}^{\mathfrak{p}}\left(1+(\varsigma-1) v_{\mathfrak{i}}^{3}\right)^{\kappa_{\mathfrak{i}}}+(\varsigma-1) \prod_{\mathfrak{i}=1}^{\mathfrak{p}}\left(1-v_{\mathfrak{i}}^{3}\right)^{\kappa_{\mathfrak{i}}}}} \\
& \otimes\left\langle\sqrt[3]{\frac{\varsigma\left\{\left(1-v_{\mathfrak{p}+1}^{3}\right)^{\kappa_{\mathfrak{p}+1}}-\left(1-v_{\mathfrak{p}+1}^{3}-\mu_{\mathfrak{p}+1}^{3}\right)^{\kappa_{\mathfrak{p}+1}}\right\}}{\left(1+(\varsigma-1) \nu_{\mathfrak{p}+1}^{3}\right)^{\kappa_{\mathfrak{p}+1}}+(\varsigma-1)\left(1-v_{\mathfrak{p}+1}^{3}\right)^{\kappa_{\mathfrak{p}+1}}}},\right\rangle \\
& \sqrt[3]{\frac{\left(1+(\varsigma-1) v_{\mathfrak{p}+1}^{3}\right)^{\kappa_{\mathfrak{p}+1}}-\left(1-v_{\mathfrak{p}+1}^{3}\right)^{\kappa_{\mathfrak{p}+1}}}{\left(1+(\varsigma-1) v_{\mathfrak{p}+1}^{3}\right)+(\varsigma-1)\left(1-v_{\mathfrak{p}+1}^{3}\right)^{\kappa_{\mathfrak{p}+1}}}} \\
& =\left\langle\sqrt[3]{\frac{\varsigma\left\{\prod_{\mathfrak{i}=1}^{\mathfrak{p}+1}\left(1-v_{\mathfrak{i}}^{3}\right)^{\kappa_{\mathfrak{i}}}-\prod_{\mathfrak{i}=1}^{\mathfrak{p}+1}\left(1-v_{\mathfrak{i}}^{3}-\mu_{\mathfrak{i}}^{3}\right)^{\kappa_{\mathfrak{i}}}\right\}}{\prod_{\mathfrak{i}=1}^{\mathfrak{p}+1}\left(1+(\varsigma-1) \nu_{\mathfrak{i}}^{3}\right)^{\kappa_{\mathfrak{i}}}+(\varsigma-1) \prod_{\mathfrak{i}=1}^{\mathfrak{p}+1}\left(1-v_{\mathfrak{i}}^{3}\right)^{\kappa_{\mathfrak{i}}}}}\right\rangle, \\
& =\left\langle\sqrt[3]{\frac{\prod_{\mathfrak{i}=1}^{\mathfrak{p}+1}\left(1+(\varsigma-1) v_{\mathfrak{i}}^{3}\right)^{\kappa_{\mathfrak{i}}}-\prod_{\mathfrak{i}=1}^{\mathfrak{p}+1}\left(1-v_{\mathfrak{i}}^{3}\right)^{\kappa_{\mathfrak{i}}}}{\prod_{\mathfrak{i}=1}^{\mathfrak{p}+1}\left(1+(\varsigma-1) \nu_{\mathfrak{i}}^{3}\right)^{\kappa_{\mathfrak{i}}}+(\varsigma-1) \prod_{\mathfrak{i}=1}^{\mathfrak{p}+1}\left(1-v_{\mathfrak{i}}^{3}\right)^{\kappa_{\mathfrak{i}}}}}\right\rangle .
\end{aligned}
$$

$\Rightarrow$ The result holds, $\forall \mathfrak{y}$.

Remark 1. Here are cases of the FFHIWGO. (i) For $\varsigma=1$, FFHIWGO becomes Fermatean fuzzy interactive weighted geometric operator (FFIWGO):

$$
\operatorname{FFIWG}\left(\mathscr{L}_{1}, \mathscr{L}_{2}, \ldots, \mathscr{L}_{\mathfrak{y}}\right)=\left\langle\sqrt[3]{\prod_{\mathfrak{i}=1}^{\mathfrak{y}}\left(1-v_{\mathfrak{i}}^{3}\right)^{\kappa_{\mathfrak{i}}}-\prod_{\mathfrak{i}=1}^{\mathfrak{y}}\left(1-v_{\mathfrak{i}}^{3}-\mu_{\mathfrak{i}}^{3}\right)^{\kappa_{\mathfrak{i}}}}, \sqrt[3]{1-\prod_{\mathfrak{i}=1}^{\mathfrak{y}}\left(1-v_{\mathfrak{i}}^{3}\right)^{\kappa_{\mathfrak{i}}}}\right\rangle .
$$


(ii) For $\varsigma=2$, FFHIWG operator becomes Fermatean fuzzy Einstein interactive weighted geometric operator (FFEIWGO):

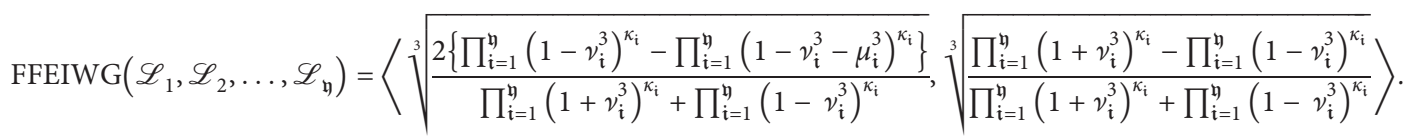

Theorem 2. The clumped value of FFSs $\mathscr{L}_{\mathfrak{i}}=\left(\mu_{\mathfrak{i}}, \nu_{\mathfrak{i}}\right)$, by using FFHIWGO, is a FFS, i.e.,

$\operatorname{FFHIWG}\left(\mathscr{L}_{1}, \mathscr{L}_{2}, \ldots, \mathscr{L}_{\mathfrak{y}}\right) \in$ FFS.

$$
\begin{aligned}
& \frac{\prod_{i=1}^{\mathfrak{y}}\left(1+(\varsigma-1) \nu_{i}^{3}\right)^{\kappa_{i}}-\prod_{i=1}^{\mathfrak{y}}\left(1-\nu_{i}^{3}\right)^{\kappa_{i}}}{\prod_{i=1}^{\mathfrak{y}}\left(1+(\varsigma-1) \nu_{i}^{3}\right)^{\kappa_{i}}+(\varsigma-1) \prod_{i=1}^{\mathfrak{y}}\left(1-v_{i}^{3}\right)^{\kappa_{i}}} \\
& =1-\frac{\varsigma \prod_{i=1}^{\mathfrak{y}}\left(1-v_{\mathfrak{i}}^{3}\right)^{\kappa_{i}}}{\prod_{\mathfrak{i}=1}^{\mathfrak{y}}\left(1+(\varsigma-1) \nu_{\mathfrak{i}}^{3}\right)^{\kappa_{\mathfrak{i}}}+(\varsigma-1) \prod_{\mathfrak{i}=1}^{\mathfrak{y}}\left(1-\nu_{\mathfrak{i}}^{3}\right)^{\kappa_{i}}} \\
& \leq 1-\prod_{\mathfrak{i}=1}^{\mathfrak{y}}\left(1-v_{\mathfrak{i}}^{3}\right)^{\kappa_{\mathfrak{i}}} \leq 1 .
\end{aligned}
$$

Also, $\left(1+(\varsigma-1) \nu_{\mathfrak{i}}^{3}\right) \geq\left(1-\nu_{\mathfrak{i}}^{3}\right) \Longrightarrow \prod_{\mathfrak{i}=1}^{\mathfrak{y}}\left(1+(\varsigma-1) \nu_{\mathfrak{i}}^{3}\right)-$ $\prod_{\mathfrak{i}=1}^{\mathfrak{y}}\left(1-v_{\mathfrak{i}}^{3}\right) \geq 0$. Therefore,

$$
\begin{gathered}
\frac{\prod_{\mathfrak{i}=1}^{\mathfrak{y}}\left(1+(\varsigma-1) v_{\mathfrak{i}}^{3}\right)^{\kappa_{\mathfrak{i}}}-\prod_{\mathfrak{i}=1}^{\mathfrak{y}}\left(1-v_{\mathfrak{i}}^{3}\right)^{\kappa_{\mathfrak{i}}}}{\prod_{\mathfrak{i}=1}^{\mathfrak{y}}\left(1+(\varsigma-1) v_{\mathfrak{i}}^{3}\right)^{\kappa_{\mathfrak{i}}}+(\varsigma-1) \prod_{\mathfrak{i}=1}^{\mathfrak{y}}\left(1-v_{\mathfrak{i}}^{3}\right)^{\kappa_{\mathfrak{i}}}} \geq 0 \\
\Rightarrow \sqrt[3]{\frac{\prod_{\mathfrak{i}=1}^{\mathfrak{y}}\left(1+(\varsigma-1) \nu_{\mathfrak{i}}^{3}\right)^{\kappa_{\mathfrak{i}}}-\prod_{\mathfrak{i}=1}^{\mathfrak{y}}\left(1-v_{\mathfrak{i}}^{3}\right)^{\kappa_{i}}}{\prod_{\mathfrak{i}=1}^{\mathfrak{y}}\left(1+(\varsigma-1) \nu_{\mathfrak{i}}^{3}\right)^{\kappa_{\mathfrak{i}}}+(\varsigma-1) \prod_{\mathfrak{i}=1}^{\mathfrak{y}}\left(1-\nu_{\mathfrak{i}}^{3}\right)^{\kappa_{\mathfrak{i}}}} \geq 0 .}
\end{gathered}
$$

Thus, $0 \leq v_{\text {FFHIWG }} \leq 1$.

Moreover,

$$
\begin{aligned}
& \frac{\varsigma\left\{\prod_{i=1}^{\mathfrak{y}}\left(1-v_{i}^{3}\right)^{\kappa_{i}}-\prod_{i=1}^{\mathfrak{y}}\left(1-v_{i}^{3}-\mu_{i}^{3}\right)^{\kappa_{i}}\right\}}{\prod_{i=1}^{\mathfrak{y}}\left(1+(\varsigma-1) \nu_{i}^{3}\right)^{\kappa_{i}}+(\varsigma-1) \prod_{i=1}^{\mathfrak{y}}\left(1-\nu_{i}^{3}\right)^{\kappa_{i}}} \\
& \leq \frac{\varsigma\left\{\prod_{i=1}^{\mathfrak{y}}\left(1-\nu_{i}^{3}\right)^{\kappa_{i}}\right\}}{\prod_{i=1}^{\mathfrak{y}}\left(1+(\varsigma-1) \nu_{i}^{3}\right)^{\kappa_{i}}+(\varsigma-1) \prod_{i=1}^{\mathfrak{y}}\left(1-\nu_{i}^{3}\right)^{\kappa_{i}}} \\
& \leq \leq \prod_{i=1}^{\mathfrak{y}}\left(1-\nu_{i}^{3}\right)^{\kappa_{i}}
\end{aligned}
$$

$\leq 1$. 
Also,

Thus, $0 \leq \mu_{\text {FFHIWG }} \leq 1$.

$$
\begin{aligned}
& \prod_{\mathfrak{i}=1}^{\mathfrak{y}}\left(1-\nu_{\mathbf{i}}^{3}\right)^{\kappa_{\mathfrak{i}}}-\prod_{\mathbf{i}=1}^{\mathfrak{y}}\left(1-\nu_{\mathfrak{i}}^{3}-\mu_{\mathfrak{i}}^{3}\right)^{\kappa_{\mathfrak{i}}} \geq 0, \\
& \frac{\varsigma\left\{\prod_{i=1}^{\mathfrak{y}}\left(1-\nu_{i}^{3}\right)^{\kappa_{i}}-\prod_{i=1}^{\mathfrak{y}}\left(1-\nu_{i}^{3}-\mu_{i}^{3}\right)^{\kappa_{i}}\right\}}{\prod_{i=1}^{\mathfrak{y}}\left(1+(\varsigma-1) \nu_{\mathfrak{i}}^{3}\right)^{\kappa_{i}}+(\varsigma-1) \prod_{i=1}^{\mathfrak{y}}\left(1-\nu_{i}^{3}\right)^{\kappa_{i}}} \geq 0,
\end{aligned}
$$

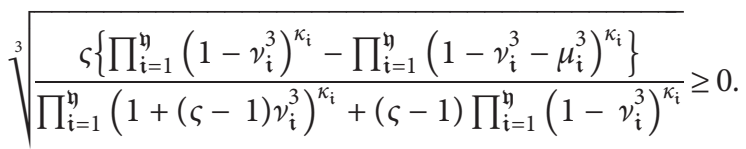

Property 1 (idempotency). If $\mathscr{L}_{\mathfrak{i}}=\mathscr{L}_{o}=\left(\mu_{o}, v_{o}\right), \forall \mathfrak{i}$, then

Proof. Since $\mathscr{L}_{\mathfrak{i}}=\mathscr{L}_{o}=\left(\mu_{o}, v_{o}\right)(\forall \mathfrak{i}=1,2, \ldots, \mathfrak{y})$ and $\sum_{\mathfrak{i}=1}^{\mathfrak{y}} \kappa_{\mathfrak{i}}=1$, by Theorem 1 ,

$$
\begin{aligned}
& \sqrt[3]{\frac{\varsigma\left\{\prod_{\mathfrak{i}=1}^{\mathfrak{y}}\left(1-v_{o}^{3}\right)^{\kappa_{\mathfrak{i}}}-\prod_{\mathfrak{i}=1}^{\mathfrak{y}}\left(1-v_{o}^{3}-\mu_{o}^{3}\right)^{\kappa_{\mathfrak{i}}}\right\}}{\prod_{\mathfrak{i}=1}^{\mathfrak{y}}\left(1+(\varsigma-1) v_{o}^{3}\right)^{\kappa_{\mathfrak{i}}}+(\varsigma-1) \prod_{\mathfrak{i}=1}^{\mathfrak{y}}\left(1-v_{o}^{3}\right)^{\kappa_{\mathfrak{i}}}}} \\
& \operatorname{FFHIWG}\left(\mathscr{L}_{1}, \mathscr{L}_{2}, \ldots, \mathscr{L}_{\mathfrak{y}}\right)=\left\langle\sqrt[3]{\frac{\prod_{i=1}^{\mathfrak{y}}\left(1+(\varsigma-1) \nu_{o}^{3}\right)^{\kappa_{\mathfrak{i}}}-\prod_{\mathfrak{i}=1}^{\mathfrak{y}}\left(1-v_{o}^{3}\right)^{\kappa_{\mathfrak{i}}}}{\prod_{\mathfrak{i}=1}^{\mathfrak{y}}\left(1+(\varsigma-1) v_{o}^{3}\right)^{\kappa_{\mathfrak{i}}}+(\varsigma-1) \prod_{\mathfrak{i}=1}^{\mathfrak{y}}\left(1-v_{o}^{3}\right)^{\kappa_{\mathfrak{i}}}}}\right\rangle \\
& =\left\langle\sqrt[3]{\frac{\varsigma\left\{\left(1-v_{o}^{3}\right)^{\sum_{\mathfrak{i}=1}^{\mathfrak{y}} \kappa_{\mathfrak{i}}}-\left(1-\nu_{o}^{3}-\mu_{o}^{3}\right)^{\sum_{\mathfrak{i}=1}^{\mathfrak{y}} \kappa_{\mathfrak{i}}}\right\}}{\left(1+(\varsigma-1) \nu_{o}^{3}\right)^{\sum_{\mathfrak{i}=1}^{\mathfrak{y}} \kappa_{\mathfrak{i}}}+(\varsigma-1)\left(1-\nu_{o}^{3}\right)^{\sum_{\mathfrak{i}=1}^{\mathfrak{y}} \kappa_{\mathfrak{i}}}}}\right\rangle \\
& \sqrt[3]{\frac{\left(1+(\varsigma-1) v_{o}^{3}\right)^{\sum_{\mathfrak{i}=1}^{\mathfrak{y}} \kappa_{\mathfrak{i}}}-\left(1-v_{o}^{3}\right)^{\sum_{\mathfrak{i}=1}^{\mathfrak{y}} \kappa_{\mathfrak{i}}}}{\left(1+(\varsigma-1) \nu_{o}^{3}\right)^{\sum_{\mathfrak{i}=1}^{\mathfrak{y}} \kappa_{\mathfrak{i}}}+(\varsigma-1)\left(1-\nu_{o}^{3}\right)^{\sum_{\mathfrak{i}=1}^{\mathfrak{y}} \kappa_{\mathfrak{i}}}}} \\
& =\left\langle\sqrt[3]{\frac{\varsigma\left\{\left(1-v_{o}^{3}\right)-\left(1-v_{o}^{3}-\mu_{o}^{3}\right)\right\}}{\left(1+(\varsigma-1) v_{o}^{3}\right)+(\varsigma-1)\left(1-v_{o}^{3}\right)}}, \sqrt[3]{\frac{\left(1+(\varsigma-1) v_{o}^{3}\right)-\left(1-v_{o}^{3}\right)}{\left(1+(\varsigma-1) v_{o}^{3}\right)+(\varsigma-1)\left(1-v_{o}^{3}\right)}}\right\rangle \\
& =\left(\mu_{o}, v_{o}\right) \text {. }
\end{aligned}
$$

Property 2 (boundedness). Let $\mathscr{L}^{-}=\left(\min _{\mathfrak{i}}\left(\mu_{\mathfrak{i}}\right), \max _{\mathfrak{i}}\left(\nu_{\mathfrak{i}}\right)\right)$ and $\mathscr{L}^{+}=\left(\max _{\mathfrak{i}}\left(\mu_{\mathfrak{i}}\right), \min _{\mathfrak{i}}\left(\nu_{\mathfrak{i}}\right)\right)$; then,

$$
\mathscr{L}^{-} \leq \operatorname{FFHIWG}\left(\mathscr{L}_{1}, \mathscr{L}_{2}, \ldots, \mathscr{L}_{\mathfrak{y}}\right) \leq \mathscr{L}^{+} \text {. }
$$

Proof. Let $f(\mathfrak{a})=(1-\mathfrak{a} / 1+(\varsigma-1) \mathfrak{a}), \mathfrak{a} \in[0,1]$; then $f^{\prime}(\mathfrak{a})=-\left(\varsigma /(1+(\varsigma-1) \mathfrak{a})^{2}\right)<0$, so $f(\mathfrak{a})$ is a decreasing function (DF). As $\nu_{\mathfrak{i}, \min }^{3} \leq \nu_{\mathfrak{i}}^{3} \leq \nu_{\mathfrak{i}, \max }^{3}, \forall \mathfrak{i}=1,2, \ldots, \mathfrak{y}$, then $f\left(\nu_{\mathfrak{i}, \text { max }}^{3}\right) \leq f\left(\nu_{\mathfrak{i}}^{3}\right) \leq f\left(\nu_{\mathfrak{i}, \text { min }}^{3}\right), \forall \mathfrak{i}$; $\quad$ that is, $\quad\left(1-v_{\mathbf{i}, \text { max }}^{3} /\right.$ 
$\left.1+(\varsigma-1) \nu_{\mathfrak{i}, \max }^{3}\right) \leq\left(1-\quad \nu_{\mathfrak{i}}^{3} / 1+(\varsigma-1) \nu_{\mathfrak{i}}^{3}\right) \leq\left(1-\nu_{\mathfrak{i}, \min }^{3} / 1+\right.$

$\left.(\varsigma-1) \nu_{\mathfrak{i}, \text { min }}^{3}\right), \forall \mathfrak{i}$. Let $\kappa_{\mathfrak{i}} \in[0,1]$ and $\sum_{\mathfrak{i}=1}^{\mathfrak{y}} \kappa_{\mathfrak{i}}=1$; we have

$$
\begin{aligned}
& \left(\frac{1-\nu_{\mathfrak{i}, \max }^{3}}{1+(\varsigma-1) \nu_{\mathfrak{i}, \text { max }}^{3}}\right)^{\kappa_{\mathfrak{i}}} \leq\left(\frac{1-\nu_{\mathfrak{i}}^{3}}{1+(\varsigma-1) \nu_{\mathfrak{i}}^{3}}\right)^{\kappa_{\mathfrak{i}}} \leq\left(\frac{1-\nu_{\mathfrak{i}, \text { min }}^{3}}{1+(\varsigma-1) \nu_{\mathfrak{i}, \min }^{3}}\right)^{\kappa_{\mathfrak{i}}} \\
& \prod_{\mathfrak{i}=1}^{\mathfrak{y}}\left(\frac{1-\nu_{\mathfrak{i}, \max }^{3}}{1+(\varsigma-1) \nu_{\mathfrak{i}, \max }^{3}}\right)^{\kappa_{\mathfrak{i}}} \leq \prod_{\mathfrak{i}=1}^{\mathfrak{y}}\left(\frac{1-\nu_{\mathfrak{i}}^{3}}{1+(\varsigma-1) \nu_{\mathfrak{i}}^{3}}\right)^{\kappa_{\mathfrak{i}}} \leq \prod_{\mathfrak{i}=1}^{\mathfrak{y}}\left(\frac{1-\nu_{\mathfrak{i}, \text { min }}^{3}}{1+(\varsigma-1) \nu_{\mathfrak{i}, \text { min }}^{3}}\right)^{\kappa_{\mathfrak{i}}}
\end{aligned}
$$

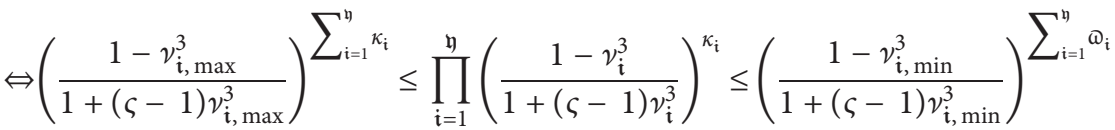

$$
\begin{aligned}
& \Leftrightarrow\left(\frac{1-v_{\mathfrak{i}, \max }^{3}}{1+(\varsigma-1) \nu_{\mathfrak{i}, \text { max }}^{3}}\right) \leq \prod_{\mathfrak{i}=1}^{\mathfrak{y}}\left(\frac{1-v_{\mathfrak{i}}^{3}}{1+(\varsigma-1) \nu_{\text {mathfraki }}^{3}}\right)^{\kappa_{\mathfrak{i}}} \leq\left(\frac{1-v_{\mathfrak{i}, \text { min }}^{3}}{1+(\varsigma-1) \nu_{\mathfrak{i}, \text { min }}^{3}}\right) \\
& \Leftrightarrow(\varsigma-1)\left(\frac{1-\nu_{\mathfrak{i}, \max }^{3}}{1+(\varsigma-1) \nu_{\mathfrak{i}, \max }^{3}}\right) \leq(\varsigma-1) \prod_{\mathfrak{i}=1}^{\mathfrak{y}}\left(\frac{1-\nu_{\mathfrak{i}}^{3}}{1+(\varsigma-1) \nu_{\mathfrak{i}}^{3}}\right)^{\omega_{\mathfrak{i}}} \leq(\varsigma-1)\left(\frac{1-\nu_{\mathfrak{i}, \min }^{3}}{1+(\varsigma-1) \nu_{\mathfrak{i}, \min }^{3}}\right) \\
& \Leftrightarrow\left(\frac{\varsigma}{1+(\varsigma-1) \nu_{\mathfrak{i}, \text { max }}^{3}}\right) \leq 1+(\varsigma-1) \prod_{\mathfrak{i}=1}^{\mathfrak{y}}\left(\frac{1-v_{\mathfrak{i}}^{3}}{1+(\varsigma-1) \nu_{\mathfrak{i}}^{3}}\right)^{\kappa_{\mathfrak{i}}} \leq\left(\frac{\varsigma}{1+(\varsigma-1) \nu_{\mathfrak{i}, \text { min }}^{3}}\right) \\
& \Leftrightarrow\left(\frac{1+(\varsigma-1) \nu_{\mathfrak{i}, \min }^{3}}{\varsigma}\right) \leq \frac{1}{1+(\varsigma-1) \prod_{\mathfrak{i}=1}^{\mathfrak{y}}\left(1-v_{\mathfrak{i}}^{3} / 1+(\varsigma-1) \nu_{\mathfrak{i}}^{3}\right)^{\kappa_{\mathfrak{i}}}} \leq\left(\frac{1+(\varsigma-1) \nu_{\mathfrak{i}, \max }^{3}}{\varsigma}\right) \\
& \Leftrightarrow\left(1+(\varsigma-1) \nu_{\mathfrak{i}, \text { min }}^{3}\right) \leq \frac{\varsigma}{1+(\varsigma-1) \prod_{\mathfrak{i}=1}^{\mathfrak{y}}\left(1-\nu_{\mathfrak{i}}^{3} / 1+(\varsigma-1) \nu_{\mathfrak{i}}^{3}\right)^{\kappa_{\mathfrak{i}}}} \leq\left(1+(\varsigma-1) v_{\mathfrak{i}, \max }^{3}\right) \\
& \Leftrightarrow(\varsigma-1) v_{\mathfrak{i}, \min }^{3} \leq \frac{\varsigma}{1+(\varsigma-1) \prod_{\mathfrak{i}=1}^{\mathfrak{y}}\left(1-v_{\mathfrak{i}}^{3} / 1+(\varsigma-1) v_{\mathfrak{i}}^{3}\right)^{\kappa_{\mathfrak{i}}}}-1 \leq(\varsigma-1) \nu_{\mathfrak{i}, \text { max }}^{3} \\
& \Leftrightarrow \nu_{\mathfrak{i}, \min }^{3} \leq \frac{\prod_{\mathfrak{i}=1}^{\mathfrak{y}}\left(1+(\varsigma-1) \nu_{\mathfrak{i}}^{3}\right)^{\kappa_{\mathfrak{i}}}-\prod_{\mathfrak{i}=1}^{\mathfrak{y}}\left(1-v_{\mathfrak{i}}^{3}\right)^{\kappa_{\mathfrak{i}}}}{\prod_{\mathfrak{i}=1}^{\mathfrak{y}}\left(1+(\varsigma-1) \nu_{\mathfrak{i}}^{3}\right)^{\Phi_{\mathfrak{i}}}+(\varsigma-1) \prod_{\mathfrak{i}=1}^{\mathfrak{y}}\left(1-v_{\mathfrak{i}}^{3}\right)^{\Phi_{\mathfrak{i}}}} \leq \nu_{\mathfrak{i}, \max }^{3} .
\end{aligned}
$$

Thus,

$$
\nu_{\mathfrak{i}, \min } \leq \sqrt[3]{\frac{\prod_{\mathfrak{i}=1}^{\mathfrak{y}}\left(1+(\varsigma-1) \nu_{\mathfrak{i}}^{3}\right)^{\kappa_{\mathfrak{i}}}-\prod_{\mathfrak{i}=1}^{\mathfrak{y}}\left(1-\nu_{\mathfrak{i}}^{3}\right)^{\kappa_{\mathfrak{i}}}}{\prod_{\mathfrak{i}=1}^{\mathfrak{y}}\left(1+(\varsigma-1) \nu_{\mathfrak{i}}^{3}\right)^{\kappa_{\mathfrak{i}}}+(\varsigma-1) \prod_{\mathfrak{i}=1}^{\mathfrak{y}}\left(1-v_{\mathfrak{i}}^{3}\right)^{\Phi_{\mathfrak{i}}}}} \leq v_{\mathfrak{i}, \max } .
$$

Consider $\quad g(\mathfrak{b})=(\varsigma-(\varsigma-1) \mathfrak{b} /(\varsigma-1) \mathfrak{b}), \mathfrak{b} \in(0,1]$, then $g^{\prime}(\mathfrak{b})=-\left(\varsigma /(\varsigma-1) \mathfrak{b}^{2}\right)$; i.e., $g(\mathfrak{b})$ is a DF on $(0,1]$. Since $1-\nu_{\mathfrak{i}, \text { max }}^{3} \leq 1-\nu_{\mathfrak{i}}^{3} \leq 1-\nu_{\mathfrak{i}, \text { min }}^{3}, \forall \dot{i}$, then $g\left(1-v_{\mathfrak{i}, \text { max }}^{3}\right) \leq$ $g\left(1-v_{\mathfrak{i}}^{3}\right) \leq g\left(1-v_{\mathfrak{i}, \max }^{3}\right), \forall \dot{i}$; that is, $\left(\varsigma-(\varsigma-1)\left(1-v_{\mathfrak{i}, \text { min }}^{3}\right.\right.$ )$\left./(\varsigma-1)\left(1-v_{\mathfrak{i}, \min }^{3}\right)\right) \leq\left(\varsigma-(\varsigma-1)\left(1-v_{\mathfrak{i}}^{3}\right) /(\varsigma-1)(1-\right.$ $\left.\left.\nu_{\mathfrak{i}}^{3}\right)\right) \leq\left(\varsigma-(\varsigma-1)\left(1-v_{\mathfrak{i}, \max }^{3}\right) /(\varsigma-1)\left(1-v_{\mathfrak{i}, \max }^{3}\right)\right)$. Then, 
8

Mathematical Problems in Engineering

$$
\begin{aligned}
& \left(\frac{\varsigma-(\varsigma-1)\left(1-v_{i, \text { min }}^{3}\right)}{(\varsigma-1)\left(1-v_{i, \text { min }}^{3}\right)}\right)^{\kappa_{i}} \leq\left(\frac{\varsigma-(\varsigma-1)\left(1-\nu_{i}^{3}\right)}{(\varsigma-1)\left(1-v_{i}^{3}\right)}\right)^{\kappa_{i}} \leq\left(\frac{\varsigma-(\varsigma-1)\left(1-v_{i, \text { max }}^{3}\right)}{(\varsigma-1)\left(1-v_{i, \text { max }}^{3}\right)}\right)^{\kappa_{i}} \\
& \prod_{\mathfrak{i}=1}^{\mathfrak{y}}\left(\frac{\varsigma-(\varsigma-1)\left(1-v_{\mathfrak{i}, \text { min }}^{3}\right)}{(\varsigma-1)\left(1-\nu_{\mathfrak{i}, \text { min }}^{3}\right)}\right)^{\kappa_{\mathfrak{i}}} \leq \prod_{\mathfrak{i}=1}^{\mathfrak{y}}\left(\frac{\varsigma-(\varsigma-1)\left(1-v_{\mathfrak{i}}^{3}\right)}{(\varsigma-1)\left(1-\nu_{\mathfrak{i}}^{3}\right)}\right)^{\kappa_{\mathfrak{i}}} \leq \prod_{\mathfrak{i}=1}^{\mathfrak{y}}\left(\frac{\varsigma-(\varsigma-1)\left(1-\nu_{\mathfrak{i}, \text { max }}^{3}\right)}{(\varsigma-1)\left(1-\nu_{\mathfrak{i}, \text { max }}^{3}\right)}\right)^{\kappa_{\mathfrak{i}}} \\
& \Rightarrow\left(\frac{\varsigma-(\varsigma-1)\left(1-v_{i, \text { min }}^{3}\right)}{(\varsigma-1)\left(1-\nu_{i, \text { min }}^{3}\right)}\right)^{\sum_{i=1}^{\mathfrak{h}} \kappa_{\mathfrak{i}}} \leq \prod_{\mathfrak{i}=1}^{\mathfrak{y}}\left(\frac{\varsigma-(\varsigma-1)\left(1-v_{i}^{3}\right)}{(\varsigma-1)\left(1-\nu_{i}^{3}\right)}\right)^{\kappa_{\mathfrak{i}}} \leq\left(\frac{\varsigma-(\varsigma-1)\left(1-\nu_{i, \text { max }}^{3}\right)}{(\varsigma-1)\left(1-\nu_{i, \text { max }}^{3}\right)}\right)^{\sum_{i=1}^{\mathfrak{y}} \kappa_{\mathfrak{i}}} \\
& \Rightarrow\left(\frac{\varsigma-(\varsigma-1)\left(1-\nu_{\mathfrak{i}, \text { min }}^{3}\right)}{(\varsigma-1)\left(1-\nu_{\mathfrak{i}, \text { min }}^{3}\right)}\right) \leq \prod_{\mathfrak{i}=1}^{\mathfrak{y}}\left(\frac{\varsigma-(\varsigma-1)\left(1-\nu_{\mathfrak{i}}^{3}\right)}{(\varsigma-1)\left(1-v_{\mathfrak{i}}^{3}\right)}\right)^{\kappa_{\mathfrak{i}}} \leq\left(\frac{\varsigma-(\varsigma-1)\left(1-\nu_{\mathfrak{i}, \text { max }}^{3}\right)}{(\varsigma-1)\left(1-\nu_{\mathfrak{i}, \text { max }}^{3}\right)}\right) \\
& \Rightarrow\left(\frac{\varsigma}{(\varsigma-1)\left(1-v_{\mathfrak{i}, \text { min }}^{3}\right)}\right) \leq \prod_{\mathfrak{i}=1}^{\mathfrak{y}}\left(\frac{\varsigma-(\varsigma-1)\left(1-v_{\mathfrak{i}}^{3}\right)}{(\varsigma-1)\left(1-v_{\mathfrak{i}}^{3}\right)}\right)^{\kappa_{\mathfrak{i}}}+1 \leq\left(\frac{\varsigma}{(\varsigma-1)\left(1-v_{\mathfrak{i}, \text { max }}^{3}\right)}\right) \\
& \Rightarrow\left(\frac{(\varsigma-1)\left(1-v_{\mathfrak{i}, \text { max }}^{3}\right)}{\varsigma}\right) \leq \frac{1}{\prod_{\mathfrak{i}=1}^{\mathfrak{y}}\left(\varsigma-(\varsigma-1)\left(1-\nu_{\mathfrak{i}}^{3}\right) /(\varsigma-1)\left(1-\nu_{\mathfrak{i}}^{3}\right)\right)^{\kappa_{\mathfrak{i}}}+1} \leq\left(\frac{(\varsigma-1)\left(1-\nu_{\mathfrak{i}, \text { min }}^{3}\right)}{\varsigma}\right) \\
& \Rightarrow\left(1-v_{\mathfrak{i}, \max }^{3}\right) \leq \frac{\varsigma}{(\varsigma-1) \prod_{\mathfrak{i}=1}^{\mathfrak{y}}\left(\varsigma-(\varsigma-1)\left(1-\nu_{\mathfrak{i}}^{3}\right) /(\varsigma-1)\left(1-\nu_{\mathfrak{i}}^{3}\right)\right)^{\kappa_{\mathfrak{i}}}+(\varsigma-1)} \leq\left(1-\nu_{\mathfrak{i}, \text { min }}^{3}\right) \\
& \Rightarrow\left(1-\nu_{\mathfrak{i}, \text { max }}^{3}\right) \leq \frac{\varsigma}{(\varsigma-1) \prod_{\mathfrak{i}=1}^{\mathfrak{y}}\left(\varsigma-(\varsigma-1) \mu_{\mathfrak{i}}^{3} /(\varsigma-1) \mu_{\mathfrak{i}}^{3}\right)^{\kappa_{\mathfrak{i}}}+(\varsigma-1)} \leq\left(1-v_{\mathfrak{i}, \text { min }}^{3}\right) .
\end{aligned}
$$

Also,

$$
\begin{aligned}
& 1-\nu_{\mathfrak{i}, \text { max }}^{3}-\mu_{\mathfrak{i}, \text { min }}^{3} \leq 1-\nu_{\mathfrak{i}}^{3}-\mu_{\mathfrak{i}}^{3} \leq 1-\nu_{\mathfrak{i}, \text { min }}^{3}-\mu_{\mathfrak{i}, \text { max }}^{3}
\end{aligned}
$$

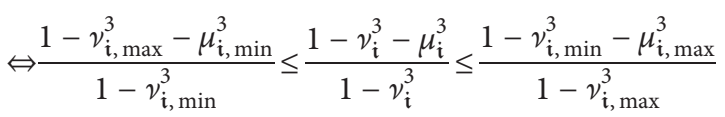

$$
\begin{aligned}
& \Leftrightarrow \frac{1-\nu_{\mathfrak{i}, \max }^{3}-\mu_{\mathfrak{i}, \min }^{3}}{1-v_{\mathfrak{i}, \min }^{3}} \leq \prod_{\mathfrak{i}=1}^{\mathfrak{y}}\left(\frac{1-\nu_{\mathfrak{i}}^{3}-\mu_{\mathfrak{i}}^{3}}{1-\nu_{\mathfrak{i}}^{3}}\right)^{\kappa_{\mathfrak{i}}} \leq \frac{1-\nu_{\mathfrak{i}, \min }^{3}-\mu_{\mathfrak{i}, \max }^{3}}{1-\nu_{\mathfrak{i}, \max }^{3}} \\
& \Leftrightarrow \frac{-v_{\mathfrak{i}, \max }^{3}+\nu_{\mathfrak{i}, \min }^{3}+\mu_{\mathfrak{i}, \max }^{3}}{1-v_{\mathfrak{i}, \max }^{3}} \leq 1-\prod_{\mathfrak{i}=1}^{\mathfrak{y}}\left(\frac{1-\nu_{\mathfrak{i}}^{3}-\mu_{\mathfrak{i}}^{3}}{1-\nu_{\mathfrak{i}}^{3}}\right)^{\kappa_{\mathfrak{i}}} \leq \frac{-\nu_{\mathfrak{i}, \min }^{3}+\nu_{\mathfrak{i}, \max }^{3}+\mu_{\mathfrak{i}, \min }^{3}}{1-\nu_{\mathfrak{i}, \min }^{3}} \\
& \Leftrightarrow-v_{\mathfrak{i}, \max }^{3}+v_{\mathfrak{i}, \text { min }}^{3}+\mu_{\mathfrak{i}, \text { max }}^{3} \leq \frac{\varsigma\left\{1-\prod_{\mathfrak{i}=1}^{\mathfrak{y}}\left(1-\nu_{\mathfrak{i}}^{3}-\mu_{\mathfrak{i}}^{3} / 1-\nu_{\mathfrak{i}}^{3}\right)^{\kappa_{\mathfrak{i}}}\right\}}{(\varsigma-1) \prod_{\mathfrak{i}=1}^{\mathfrak{y}}\left(1+(\varsigma-1) \nu_{\mathfrak{i}}^{3} /(\varsigma-1)\left(1-\nu_{\mathfrak{i}}^{3}\right)\right)^{\kappa_{\mathfrak{i}}}+(\varsigma-1)} \leq-v_{\mathfrak{i}, \text { min }}^{3}+v_{\mathfrak{i}, \text { max }}^{3}+\mu_{\mathfrak{i}, \text { min }}^{3} \\
& \Leftrightarrow \mu_{\mathfrak{i}, \max }^{3} \leq \frac{\varsigma\left\{\prod_{\mathfrak{i}=1}^{\mathfrak{y}}\left(1-\nu_{\mathfrak{i}}^{3}\right)^{\kappa_{\mathfrak{i}}}-\prod_{\mathfrak{i}=1}^{\mathfrak{y}}\left(1-\nu_{\mathfrak{i}}^{3}-\mu_{\mathfrak{i}}^{3}\right)^{\kappa_{\mathfrak{i}}}\right\}}{\prod_{\mathfrak{i}=1}^{\mathfrak{y}}\left(1+(\varsigma-1) \nu_{\mathfrak{i}}^{3}\right)^{\kappa_{\mathfrak{i}}}+(\varsigma-1) \prod_{\mathfrak{i}=1}^{\mathfrak{y}}\left(1-v_{\mathfrak{i}}^{3}\right)^{\kappa_{\mathfrak{i}}}} \leq \mu_{\mathfrak{i}, \text { min }}^{3} \\
& \Leftrightarrow \mu_{i, \max } \leq \sqrt[3]{\frac{\varsigma\left\{\prod_{\mathfrak{i}=1}^{\mathfrak{y}}\left(1-v_{\mathfrak{i}}^{3}\right)^{\kappa_{\mathfrak{i}}}-\prod_{\mathfrak{i}=1}^{\mathfrak{y}}\left(1-v_{\mathfrak{i}}^{3}-\mu_{\mathfrak{i}}^{3}\right)^{\kappa_{\mathfrak{i}}}\right\}}{\prod_{\mathfrak{i}=1}^{\mathfrak{y}}\left(1+(\varsigma-1) \nu_{\mathfrak{i}}^{3}\right)^{\kappa_{\mathfrak{i}}}+(\varsigma-1) \prod_{\mathfrak{i}=1}^{\mathfrak{y}}\left(1-v_{\mathfrak{i}}^{3}\right)^{\kappa_{\mathfrak{i}}}}} \leq \mu_{\mathfrak{i}, \min } .
\end{aligned}
$$


Let $\operatorname{FFHIWG}\left(\mathscr{L}_{1}, \mathscr{L}_{2}, \ldots, \mathscr{L}_{\mathfrak{n}}\right)=\mathscr{L}=\left\langle\mu_{\mathscr{L}}, v_{\mathscr{L}}\right\rangle$; then, from inequalities (20) and (22), $\mu_{\min } \leq \mu_{\mathscr{L}} \leq \mu_{\max }, v_{\max } \leq$ $v_{\mathscr{L}} \leq \nu_{\text {min }}$, where $\mu_{\text {min }}=\min _{\mathfrak{i}}\left\{\mu_{\mathfrak{i}}\right\}, \mu_{\max }=\max _{\mathfrak{i}}\left\{\mu_{\mathfrak{i}}\right\}, v_{\text {min }}=$ $\min _{\mathfrak{i}}\left\{v_{\mathfrak{i}}\right\}$, and $v_{\max }=\max _{\mathfrak{i}}\left\{v_{\mathfrak{i}}\right\}$. So, $S(\mathscr{L})=\mu_{\mathscr{L}}^{3}-v_{\mathscr{L}}^{3} \leq$ $\mu_{\max ^{3}}-v_{\max ^{3}}=S\left(\mathscr{L}^{+}\right) \quad$ and $\quad S(\mathscr{L})=\mu_{\mathscr{L}}^{3}-v_{\mathscr{L}}^{3} \geq \mu_{\min ^{3}-}$ $v_{\min ^{3}}=S\left(\mathscr{L}^{-}\right)$. As $S(\mathscr{L})<S\left(\mathscr{L}^{+}\right)$and $S(\mathscr{L})>S\left(\mathscr{L}^{-}\right)$,

$$
\mathscr{L}^{-} \leq \operatorname{FFHIWG}\left(\mathscr{L}_{1}, \mathscr{L}_{2}, \ldots, \mathscr{L}_{\mathfrak{n}}\right) \leq \mathscr{L}^{+} \text {. }
$$

Property 3 (monotonicity). If $\mathscr{L}_{\mathfrak{i}} \leq \mathscr{T}_{\mathfrak{i}}, \forall \mathfrak{i}$, then
$\operatorname{FFHIWG}\left(\mathscr{L}_{1}, \mathscr{L}_{2}, \ldots, \mathscr{L}_{\mathfrak{y}}\right) \leq \operatorname{FFHIWG}\left(\mathscr{T}_{1}, \mathscr{T}_{2}, \ldots, \mathscr{T}_{\mathfrak{y}}\right)$.

Proof. It can be proved on similar lines to the above.

Property 4 (shift iInvariance). If $\mathscr{T}=\left(\mu_{\mathscr{T}}, v_{\mathscr{T}}\right)$ is another FFS, then

$$
\operatorname{FFHIWG}\left(\mathscr{L}_{1} \otimes \mathscr{T}, \mathscr{L}_{2} \otimes \mathscr{T}, \ldots, \mathscr{L}_{\mathfrak{y}} \otimes \mathscr{T}\right)=\operatorname{FFHIWG}\left(\mathscr{L}_{1}, \mathscr{L}_{2}, \ldots, \mathscr{L}_{\mathfrak{\eta}}\right) \otimes \mathscr{T}
$$

Proof. As $\mathscr{L}_{\mathfrak{i}}, \mathscr{T} \in$ FFSs, so

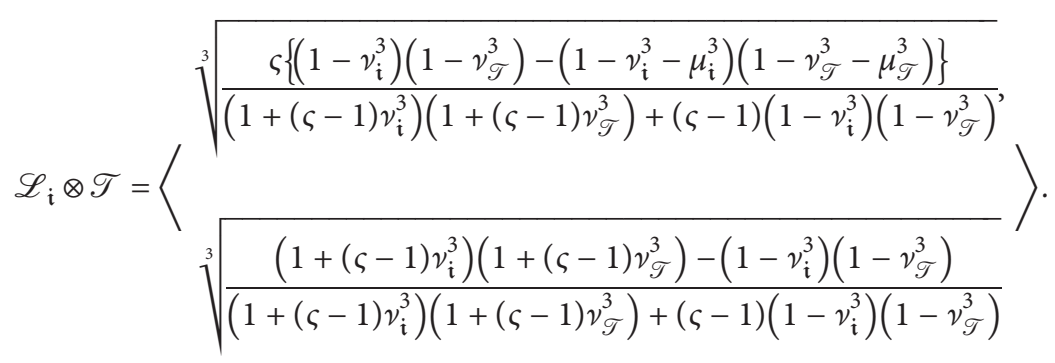

Therefore,

$$
\begin{aligned}
& \operatorname{FFHIWG}\left(\mathscr{L}_{1} \otimes \mathscr{T}, \mathscr{L}_{2} \otimes \mathscr{T}, \ldots, \mathscr{L}_{\mathfrak{y}} \otimes \mathscr{T}\right)
\end{aligned}
$$

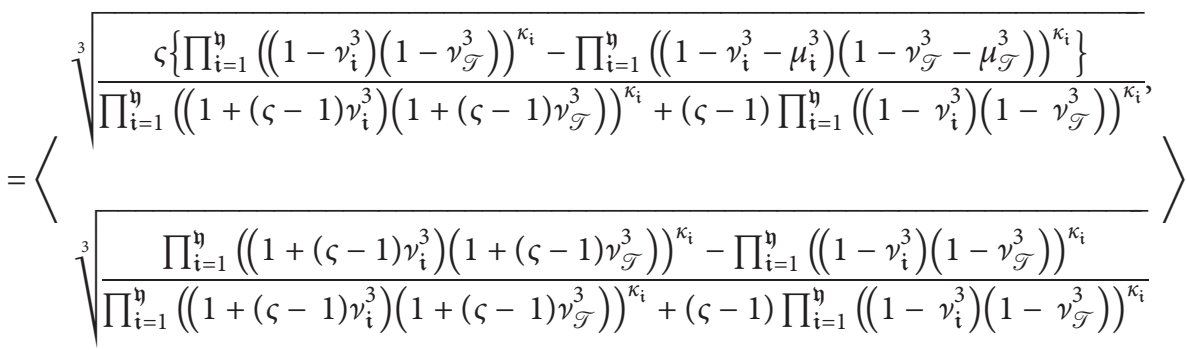

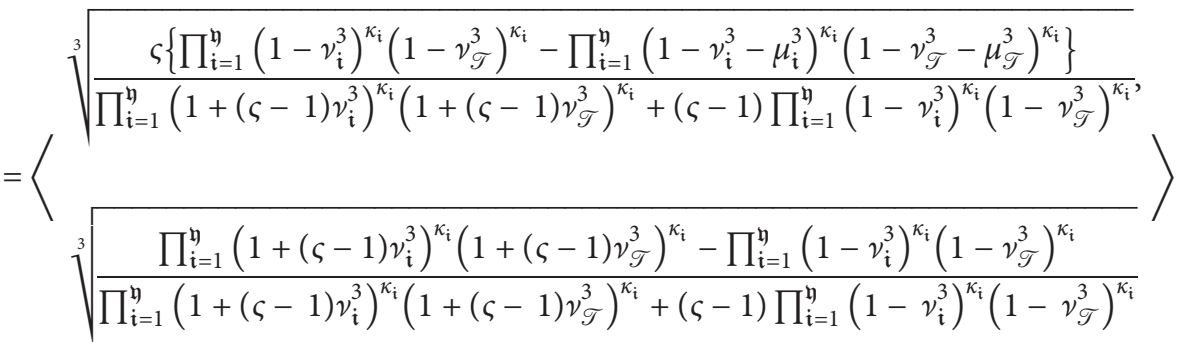




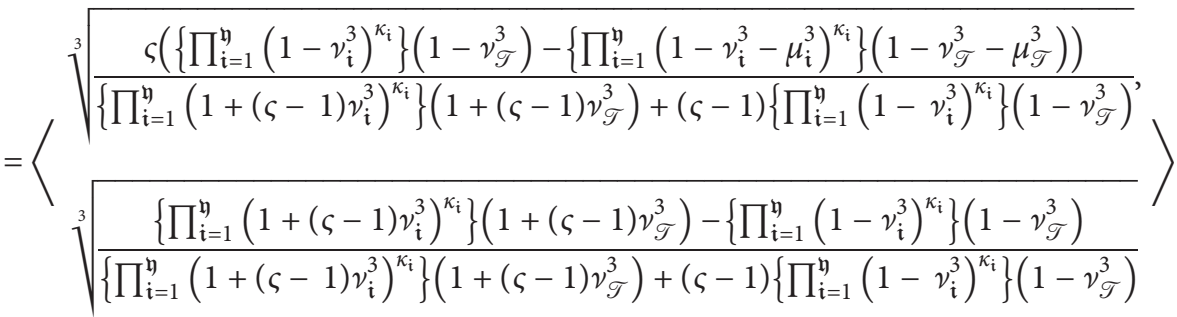

$$
\begin{aligned}
& =\operatorname{FFHIWG}\left(\mathscr{L}_{1}, \mathscr{L}_{2}, \ldots, \mathscr{L}_{\mathfrak{y}}\right) \otimes \mathscr{T} \text {. }
\end{aligned}
$$

Property 5 (homogeneity). Let $\lambda>0$, then

Proof. Since $\mathscr{L}_{\mathfrak{i}}=\left(\mu_{\mathfrak{i}}, \nu_{\mathfrak{i}}\right)$ are FFSs and $\lambda>0$, therefore $\operatorname{FFHIWG}\left(\lambda \mathscr{L}_{1}, \lambda \mathscr{L}_{2}, \ldots, \lambda \mathscr{L}_{\mathfrak{\eta}}\right)=\lambda \operatorname{FFHIWG}\left(\mathscr{L}_{1}, \mathscr{L}_{2}, \ldots, \mathscr{L}_{\mathfrak{\eta}}\right)$.

$$
\lambda \mathscr{L}_{\mathfrak{i}}=\left\langle\sqrt[3]{\frac{\varsigma\left\{\left(1-\nu^{3}\right)^{\lambda}-\left(1-\nu^{3}-\mu^{3}\right)^{\lambda}\right\}}{\left(1+(\varsigma-1) \nu^{3}\right)^{\lambda}+(\varsigma-1)\left(1-\nu^{3}\right)^{\lambda}}}, \sqrt[3]{\frac{\left(1+(\varsigma-1) \nu^{3}\right)^{\lambda}-\left(1-\nu^{3}\right)^{\lambda}}{\left(1+(\varsigma-1) \nu^{3}\right)^{\lambda}+(\varsigma-1)\left(1-\nu^{3}\right)^{\lambda}}}\right\rangle .
$$

Therefore,

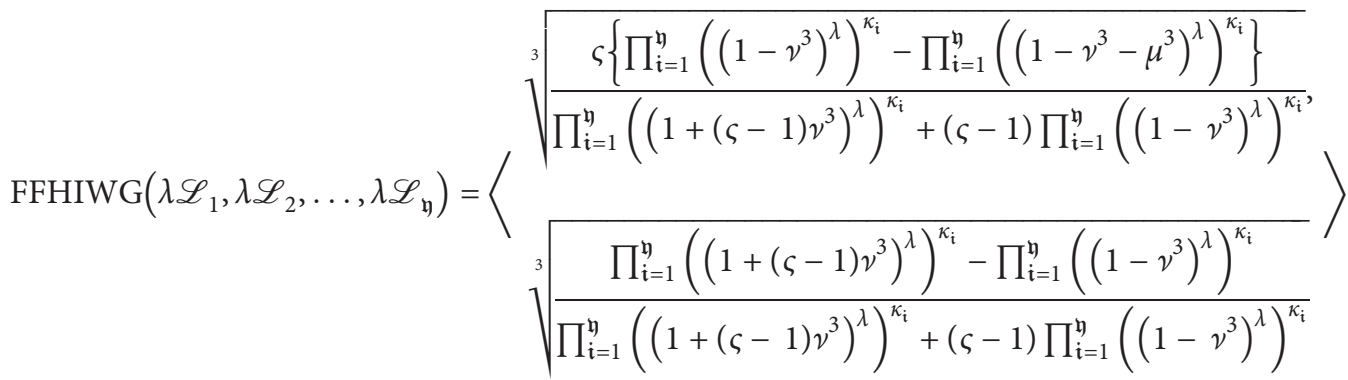

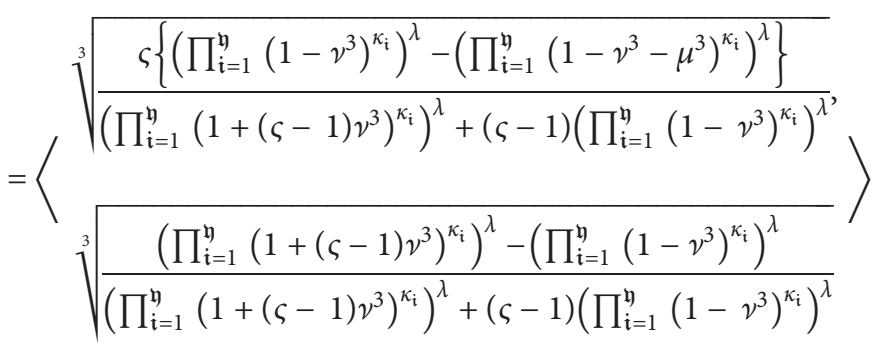

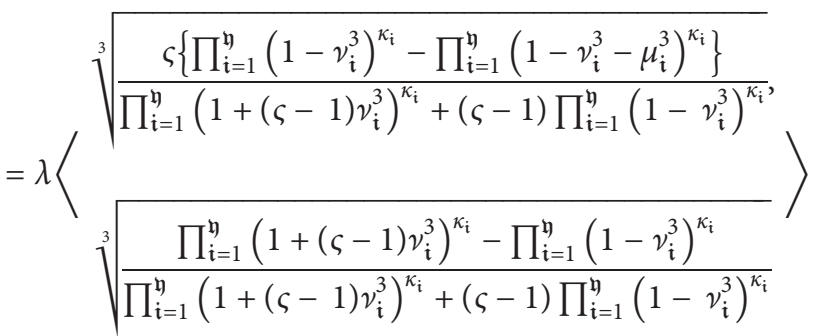

$$
\begin{aligned}
& =\lambda \operatorname{FFHIWG}\left(\mathscr{L}_{1}, \mathscr{L}_{2}, \ldots, \mathscr{L}_{\mathfrak{\eta}}\right) \text {. }
\end{aligned}
$$


Property 6. Let $\mathscr{L}_{\mathfrak{i}}=\left(\mu_{\mathscr{L}_{i}}, v_{\mathscr{L}_{\mathfrak{i}}}\right)$ and $\mathscr{T}_{\mathfrak{i}}=\left(\mu_{\mathscr{T}_{\mathfrak{i}}}, \nu_{\mathscr{T}_{\mathfrak{i}}}\right)$ be two collections of FFSs; then

$\operatorname{FFHIWG}\left(\mathscr{L}_{1} \otimes \mathscr{T}_{1}, \mathscr{L}_{2} \otimes \mathscr{T}_{2}, \ldots, \mathscr{L}_{\mathfrak{y}} \otimes \mathscr{T}_{\mathfrak{y}}\right)=\operatorname{FFHIWG}\left(\mathscr{L}_{1}, \mathscr{L}_{2}, \ldots, \mathscr{L}_{\mathfrak{y}}\right) \otimes \operatorname{FFHIWG}\left(\mathscr{T}_{1}, \mathscr{T}_{2}, \ldots, \mathscr{T}_{\mathfrak{y}}\right)$

Proof. As $\mathscr{L}_{i}=\left(\mu_{\mathscr{L}_{i}}, v_{\mathscr{L}_{i}}\right)$ and $\mathscr{T}_{i}=\left(\mu_{\mathscr{T}_{i}}, v_{\mathscr{T}_{i}}\right)$ are two collections of FFSs, then

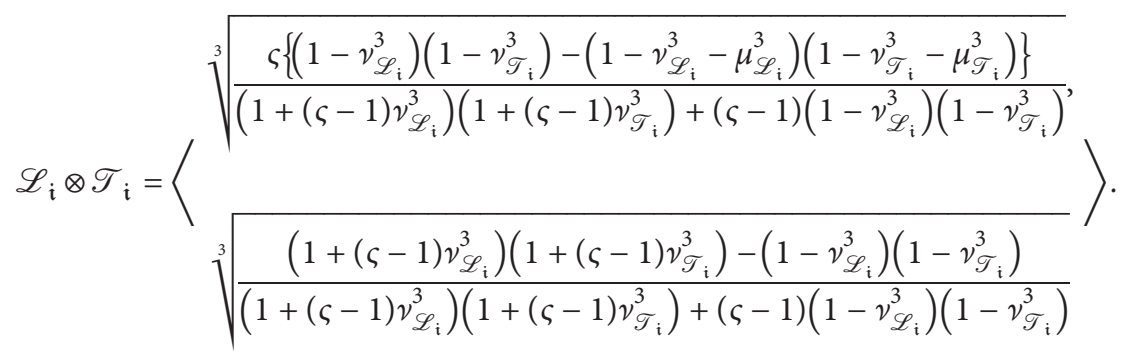

Therefore,

$\operatorname{FFHIWG}\left(\mathscr{L}_{1} \otimes \mathscr{T}_{1}, \mathscr{L}_{2} \otimes \mathscr{T}_{2}, \ldots, \mathscr{L}_{\mathfrak{y}} \otimes \mathscr{T}_{\mathfrak{y}}\right)$

$$
\begin{aligned}
& \sqrt[3]{\frac{\varsigma\left\{\prod_{\mathfrak{i}=1}^{\mathfrak{y}}\left(\left(1-v_{\mathscr{L}_{i}}^{3}\right)\left(1-\nu_{\mathscr{T}_{\mathfrak{i}}}^{3}\right)\right)^{\kappa_{\mathfrak{i}}}-\prod_{\mathfrak{i}=1}^{\mathfrak{y}}\left(\left(1-v_{\mathscr{L}_{\mathfrak{i}}}^{3}-\mu_{\mathscr{L}_{i}}^{3}\right)\left(1-\nu_{\mathscr{T}_{\mathfrak{i}}}^{3}-\mu_{\mathscr{T}_{\mathfrak{i}}}^{3}\right)\right)^{\kappa_{\mathfrak{i}}}\right\}}{\prod_{\mathfrak{i}=1}^{\mathfrak{y}}\left(\left(1+(\varsigma-1) \nu_{\mathscr{L}_{\mathfrak{i}}}^{3}\right)\left(1+(\varsigma-1) \nu_{\mathscr{T}_{\mathfrak{i}}}^{3}\right)\right)^{\kappa_{\mathfrak{i}}}+(\varsigma-1) \prod_{\mathfrak{i}=1}^{\mathfrak{y}}\left(\left(1-\nu_{\mathscr{L}_{\mathfrak{i}}}^{3}\right)\left(1-v_{\mathscr{T}_{\mathfrak{i}}}^{3}\right)\right)^{\kappa_{\mathfrak{i}}}},} \\
& =\left\langle\sqrt[3]{\frac{\prod_{\mathfrak{i}=1}^{\mathfrak{y}}\left(\left(1+(\varsigma-1) v_{\mathscr{L}_{\mathfrak{i}}}^{3}\right)\left(1+(\varsigma-1) \nu_{\mathscr{T}_{\mathfrak{i}}}^{3}\right)\right)^{\kappa_{\mathfrak{i}}}-\prod_{\mathfrak{i}=1}^{\mathfrak{y}}\left(\left(1-v_{\mathscr{L}_{\mathfrak{i}}}^{3}\right)\left(1-v_{\mathscr{T}_{\mathfrak{i}}}^{3}\right)\right)^{\kappa_{\mathfrak{i}}}}{\prod_{\mathfrak{i}=1}^{\mathfrak{y}}\left(\left(1+(\varsigma-1) \nu_{\mathscr{L}_{\mathfrak{i}}}^{3}\right)\left(1+(\varsigma-1) \nu_{\mathscr{T}_{\mathfrak{i}}}^{3}\right)\right)^{\kappa_{\mathfrak{i}}}+(\varsigma-1) \prod_{\mathfrak{i}=1}^{\mathfrak{y}}\left(\left(1-v_{\mathscr{L}_{\mathfrak{i}}}^{3}\right)\left(1-v_{\mathscr{T}_{\mathfrak{i}}}^{3}\right)\right)^{\kappa_{\mathfrak{i}}}}}\right\rangle
\end{aligned}
$$

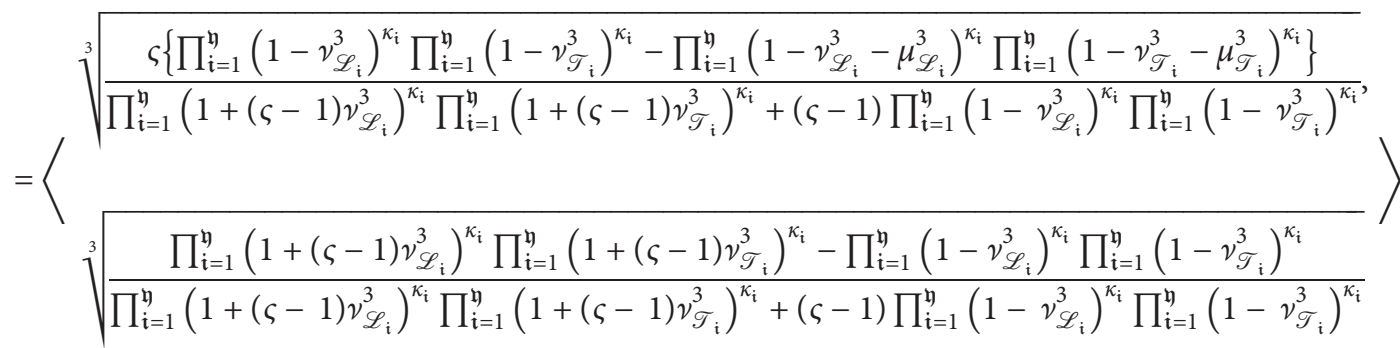

$$
=\left\langle\sqrt[3]{\frac{\varsigma\left\{\prod_{\mathfrak{i}=1}^{\mathfrak{y}}\left(1-v_{\mathscr{L}_{i}}^{3}\right)^{\kappa_{\mathfrak{i}}}-\prod_{\mathfrak{i}=1}^{\mathfrak{y}}\left(1-v_{\mathscr{L}_{\mathfrak{i}}}^{3}-\mu_{\mathscr{L}_{i}}^{3}\right)^{\kappa_{\mathfrak{i}}}\right\}}{\prod_{\mathfrak{i}=1}^{\mathfrak{y}}\left(1+(\varsigma-1) \nu_{\mathscr{L}_{\mathfrak{i}}}^{3}\right)^{\kappa_{\mathfrak{i}}}+(\varsigma-1) \prod_{\mathfrak{i}=1}^{\mathfrak{y}}\left(1-v_{\mathscr{L}_{\mathfrak{i}}}^{3}\right)^{\kappa_{\mathfrak{i}}}}}, \sqrt[3]{\frac{\prod_{\mathfrak{i}=1}^{\mathfrak{y}}\left(1+(\varsigma-1) \nu_{\mathscr{L}_{\mathfrak{i}}}^{3}\right)^{\kappa_{\mathfrak{i}}}-\prod_{\mathfrak{i}=1}^{\mathfrak{y}}\left(1-v_{\mathscr{L}_{\mathfrak{i}}}^{3}\right)^{\kappa_{\mathfrak{i}}}}{\prod_{\mathfrak{i}=1}^{\mathfrak{y}}\left(1+(\varsigma-1) v_{\mathscr{L}_{\mathfrak{i}}}^{3}\right)^{\kappa_{\mathfrak{i}}}+(\varsigma-1) \prod_{\mathfrak{i}=1}^{\mathfrak{y}}\left(1-v_{\mathscr{L}_{i}}^{3}\right)^{\kappa_{\mathfrak{i}}}}}\right\rangle
$$$$
\otimes\left\langle\sqrt[3]{\frac{\varsigma\left\{\prod_{\mathfrak{i}=1}^{\mathfrak{y}}\left(1-v_{\mathscr{T}_{\mathfrak{i}}}^{3}\right)^{\kappa_{\mathfrak{i}}}-\prod_{\mathfrak{i}=1}^{\mathfrak{y}}\left(1-v_{\mathscr{T}_{\mathfrak{i}}}^{3}-\mu_{\mathscr{T}_{i}}^{3}\right)^{\kappa_{\mathfrak{i}}}\right\}}{\prod_{\mathfrak{i}=1}^{\mathfrak{y}}\left(1+(\varsigma-1) \nu_{\mathscr{T}_{\mathfrak{i}}}^{3}\right)^{\kappa_{\mathfrak{i}}}+(\varsigma-1) \prod_{\mathfrak{i}=1}^{\mathfrak{y}}\left(1-v_{\mathscr{T}_{\mathfrak{i}}}^{3}\right)^{\kappa_{\mathfrak{i}}}}}, \sqrt[3]{\frac{\prod_{\mathfrak{i}=1}^{\mathfrak{y}}\left(1+(\varsigma-1) \nu_{\mathscr{T}_{\mathfrak{i}}}^{3}\right)^{\kappa_{\mathfrak{i}}}-\prod_{\mathfrak{i}=1}^{\mathfrak{y}}\left(1-v_{\mathscr{T}_{i}}^{3}\right)^{\kappa_{\mathfrak{i}}}}{\prod_{\mathfrak{i}=1}^{\mathfrak{y}}\left(1+(\varsigma-1) \nu_{\mathscr{T}_{\mathfrak{i}}}^{3}\right)^{\kappa_{\mathfrak{i}}}+(\varsigma-1) \prod_{\mathfrak{i}=1}^{\mathfrak{y}}\left(1-v_{\mathscr{T}_{\mathfrak{i}}}^{3}\right)^{\kappa_{\mathfrak{i}}}}}\right\rangle
$$$$
=\operatorname{FFHIWG}\left(\mathscr{L}_{1}, \mathscr{L}_{2}, \ldots, \mathscr{L}_{\mathfrak{y}}\right) \otimes \operatorname{FFHIWG}\left(\mathscr{T}_{1}, \mathscr{T}_{2}, \ldots, \mathscr{T}_{\mathfrak{y}}\right) .
$$ 
Property 7. Let $\mathscr{L}_{\mathfrak{i}}=\left(\mu_{\mathfrak{i}}, \nu_{\mathfrak{i}}\right)$ and $\mathscr{T}=(\mu, \nu)$ be FFSs and $\eta>0$; then

$$
\operatorname{FFHIWG}\left(\eta \mathscr{L}_{1} \otimes \mathscr{T}, \eta \mathscr{L}_{2} \otimes \mathscr{T}, \ldots, \eta \mathscr{L}_{\mathfrak{y}} \otimes \mathscr{T}\right)=\eta \operatorname{FFHIWG}\left(\mathscr{L}_{1}, \mathscr{L}_{2}, \ldots, \mathscr{L}_{\mathfrak{y}}\right) \otimes \mathscr{T}
$$

Proof. By applying Properties 1, 5, and 6, we can prove it.

\section{Fermatean Fuzzy HIOWG Operators}

This section elaborates the notion of Hamacher interactive ordered weighted geometric operators (HIOWGO) under FFSs.
Definition 7. Let $\mathscr{L}_{\mathfrak{i}}=\left(\mu_{\mathfrak{i}}, \nu_{\mathfrak{i}}\right)$ be a collection of FFSs and $\kappa=\left(\kappa_{1}, \kappa_{2}, \ldots, \kappa_{\mathfrak{\eta}}\right)^{T}$ be its WV, such that $\kappa_{\mathfrak{i}}>0$ and $\sum_{\mathfrak{i}=1}^{\mathfrak{y}} \kappa_{\mathfrak{i}}=1$; then FFHIOWG: $\Omega^{\mathfrak{y}} \longrightarrow \Omega$ is defined as

$$
\operatorname{FFHIWG}\left(\mathscr{L}_{1}, \mathscr{L}_{2}, \ldots, \mathscr{L}_{\mathfrak{y}}\right)=\kappa_{1} \mathscr{L}_{\varrho(1)} \otimes \kappa_{2} \mathscr{L}_{\varrho(2)} \otimes \cdots \kappa_{\mathfrak{y}} \mathscr{L}_{\varrho(\mathfrak{y})},
$$

where $(\varrho(1), \varrho(2), \ldots, \varrho(\mathfrak{y}))$ is a permutation of Theorem 3. Let $\mathscr{L}_{\mathfrak{i}}=\left(\mu_{\mathfrak{i}}, \nu_{\mathfrak{i}}\right)$ be a collection of FFSs; then $(1,2, \ldots, \mathfrak{y})$, such that $\varrho(\mathfrak{i}-1) \geq \varrho(\mathfrak{i})$ for any $\mathfrak{i}$.

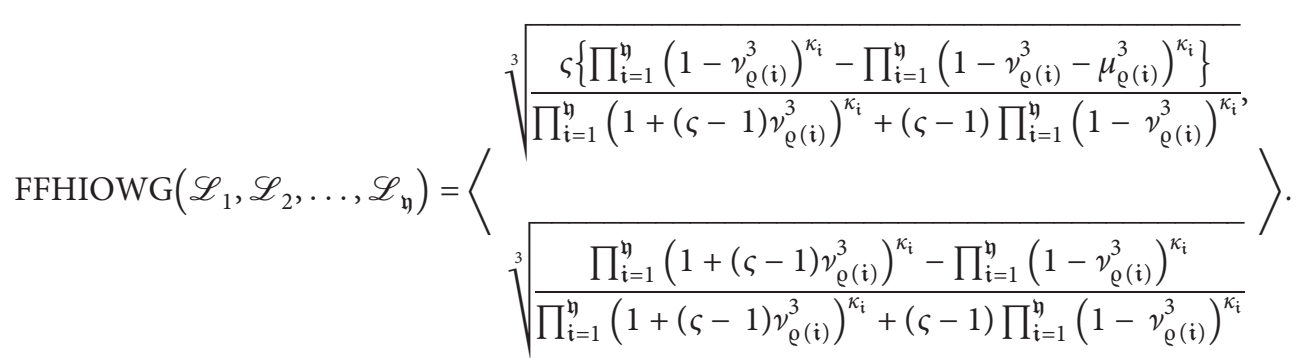

Proof. The proof is similar to that of Theorem 1.

Remark 2. The two cases of the FFHIOWG operator are as follows: (i) For $\varsigma=1$, FFHIOWGO becomes FF interactive ordered weighted geometric operator (FFIOWGO):

$$
\operatorname{FFIOWG}\left(\mathscr{L}_{1}, \mathscr{L}_{2}, \ldots, \mathscr{L}_{\mathfrak{y}}\right)=\left\langle\sqrt[3]{\prod_{\mathfrak{i}=1}^{\mathfrak{y}}\left(1-v_{\varrho(\mathfrak{i})}^{3}\right)^{\kappa_{\mathfrak{i}}}-\prod_{\mathfrak{i}=1}^{\mathfrak{y}}\left(1-v_{\varrho(\mathfrak{i})}^{3}-\mu_{\varrho(\mathfrak{i})}^{3}\right)^{\kappa_{\mathfrak{i}}}}, \sqrt[3]{1-\prod_{\mathfrak{i}=1}^{\mathfrak{y}}\left(1-v_{\varrho(\mathfrak{i})}^{3}\right)^{\kappa_{\mathfrak{i}}}}\right\rangle .
$$

(ii) For $\varsigma=2$, FFHIOWGO becomes FF Einstein interactive ordered weighted geometric operator (FFEIOWGO) operator: 


$$
\operatorname{FFEIOWG}\left(\mathscr{L}_{1}, \mathscr{L}_{2}, \ldots, \mathscr{L}_{\mathfrak{y}}\right)=\left\langle\frac{\sqrt[3]{\frac{2\left\{\prod_{i=1}^{\mathfrak{y}}\left(1-v_{\varrho(i)}^{3}\right)^{\kappa_{i}}-\prod_{i=1}^{\mathfrak{y}}\left(1-v_{\varrho(i)}^{3}-\mu_{\varrho(i)}^{3}\right)^{\kappa_{i}}\right\}}{\prod_{\mathfrak{i}=1}^{\mathfrak{y}}\left(1+v_{\varrho(i)}^{3}\right)^{\kappa_{i}}+\prod_{\mathfrak{i}=1}^{\mathfrak{y}}\left(1-v_{\varrho(i)}^{3}\right)^{\kappa_{i}}}}}{\sqrt[3]{\frac{\prod_{i=1}^{\mathfrak{y}}\left(1+v_{\varrho(i)}^{3}\right)^{\kappa_{i}}-\prod_{i=1}^{\mathfrak{y}}\left(1-v_{\varrho(i)}^{3}\right)^{\kappa_{i}}}{\prod_{i=1}^{\mathfrak{y}}\left(1+v_{\varrho(i)}^{3}\right)^{\kappa_{i}}+\prod_{i=1}^{\mathfrak{y}}\left(1-v_{\varrho(i)}^{3}\right)^{\kappa_{i}}}}}\right\rangle .
$$

Property 8. Let $\mathscr{L}_{\mathfrak{i}}=\left(\mu_{\mathfrak{i}}, \nu_{\mathfrak{i}}\right)$ be a collection of FFSs and $\kappa=$ $\left(\kappa_{1}, \kappa_{2}, \ldots, \kappa_{\mathfrak{y}}\right)^{T}$ its WV such that $\kappa_{\mathfrak{i}}>0$ and $\sum_{\mathfrak{i}=1}^{\mathfrak{y}} \kappa_{\mathfrak{i}}=1$.

(i) Idempotency: if $\mathscr{L}_{\mathfrak{i}}=\mathscr{L}_{o}=\left(\mu_{o}, v_{o}\right), \forall \mathfrak{i}$, then

$$
\operatorname{FFHIOWG}\left(\mathscr{L}_{1}, \mathscr{L}_{2}, \ldots, \mathscr{L}_{\mathfrak{y}}\right)=\mathscr{L}_{\text {o }} \text {. }
$$

(ii) Boundedness: let $\mathscr{L}^{-}=\left(\min _{\mathfrak{i}}\left(\mu_{\mathfrak{i}}\right), \max _{\mathfrak{i}}\left(\nu_{\mathfrak{i}}\right)\right)$ and $\mathscr{L}^{+}=\left(\max _{\mathfrak{i}}\left(\mu_{\mathfrak{i}}\right), \min _{\mathfrak{i}}\left(\nu_{\mathfrak{i}}\right)\right)$; then

$$
\mathscr{L}^{-} \leq \operatorname{FFHIOWG}\left(\mathscr{L}_{1}, \mathscr{L}_{2}, \ldots, \mathscr{L}_{\mathfrak{n}}\right) \leq \mathscr{L}^{+} \text {. }
$$

(iii) Monotonicity: when $\mathscr{L}_{\mathfrak{i}} \leq \mathscr{T}_{\mathfrak{i}}, \forall \mathfrak{i}$, then

$$
\operatorname{FFHIOWG}\left(\mathscr{L}_{1}, \mathscr{L}_{2}, \ldots, \mathscr{L}_{\mathfrak{y}}\right) \leq \operatorname{FFHIOWG}\left(\mathscr{T}_{1}, \mathscr{T}_{2}, \ldots, \mathscr{T}_{\mathfrak{y}}\right) \text {. }
$$

(iv) Shift invariance: if $\mathscr{T}=\left(\mu_{\mathscr{T}}, v_{\mathscr{T}}\right)$ is another FFS, then

$$
\operatorname{FFHIOWG}\left(\mathscr{L}_{1} \otimes \mathscr{T}, \mathscr{L}_{2} \otimes \mathscr{T}, \ldots, \mathscr{L}_{\mathfrak{y}} \otimes \mathscr{T}\right)=\operatorname{FFHIOWG}\left(\mathscr{L}_{1}, \mathscr{L}_{2}, \ldots, \mathscr{L}_{\mathfrak{n}}\right) \otimes \mathscr{T}
$$

(v) Homogeneity: let $\lambda>0$; then,

$$
\operatorname{FFHIOWG}\left(\lambda \mathscr{L}_{1}, \lambda \mathscr{L}_{2}, \ldots, \lambda \mathscr{L}_{\mathfrak{n}}\right)=\lambda \operatorname{FFHIOWG}\left(\mathscr{L}_{1}, \mathscr{L}_{2}, \ldots, \mathscr{L}_{\mathfrak{n}}\right)
$$

Proof. The proof is similar to that for FFHIWG operator.

\section{Fermatean Fuzzy Hamacher Interactive Hybrid Weighted Geometric Operators}

Definition 8. Let $\mathscr{L}_{\mathfrak{i}}=\left(\mu_{\mathfrak{i}}, \nu_{\mathfrak{i}}\right)$ be a collection of FFSs; then, FFHIHWG: $\Omega^{\mathfrak{y}} \longrightarrow \Omega$ is defined as

$$
\operatorname{FFHIHWG}\left(\mathscr{L}_{1}, \mathscr{L}_{2}, \ldots, \mathscr{L}_{\mathfrak{n}}\right)=\kappa_{1} \dot{\mathscr{L}}_{1} \otimes \kappa_{2} \dot{\mathscr{L}}_{2} \otimes \cdots \otimes \kappa_{\mathfrak{y}} \dot{\mathscr{L}}_{\mathfrak{y}},
$$

where 'WV' associated with FFHIHWG is $\kappa=\left(\kappa_{1}, \kappa_{2}, \ldots, \kappa_{\mathfrak{n}}\right)^{T}$ and $\phi=\left(\phi_{1}, \phi_{2}, \ldots, \phi_{\mathfrak{n}}\right)^{T}$ is the WV of $\mathscr{L}_{\mathfrak{i}}$, such that $\phi_{\mathfrak{i}} \in u 0,1$ and $\sum_{\mathfrak{i}=1}^{\mathfrak{y}} \phi_{\mathfrak{i}}=1$. Consider $\dot{\mathscr{L}}$ is the $i$ th largest among all the weighted FFSs $\left(\mathscr{L}=\mathfrak{y} \phi_{\mathfrak{i}} \mathscr{L}_{\mathfrak{i}}\right)$ and $(\varrho(1), \varrho(2), \ldots, \varrho(\mathfrak{y}))$ is a permutation of $(1,2, \ldots, \mathfrak{y})$, such that $\varrho(\mathfrak{i}-1) \geq \varrho(\mathfrak{i})$ for any $\mathfrak{i}$.

Theorem 4. Let $\mathscr{L}_{\mathfrak{i}}=\left(\mu_{\mathfrak{i}}, \nu_{\mathfrak{i}}\right)$ be a collection of FFSs; then, 


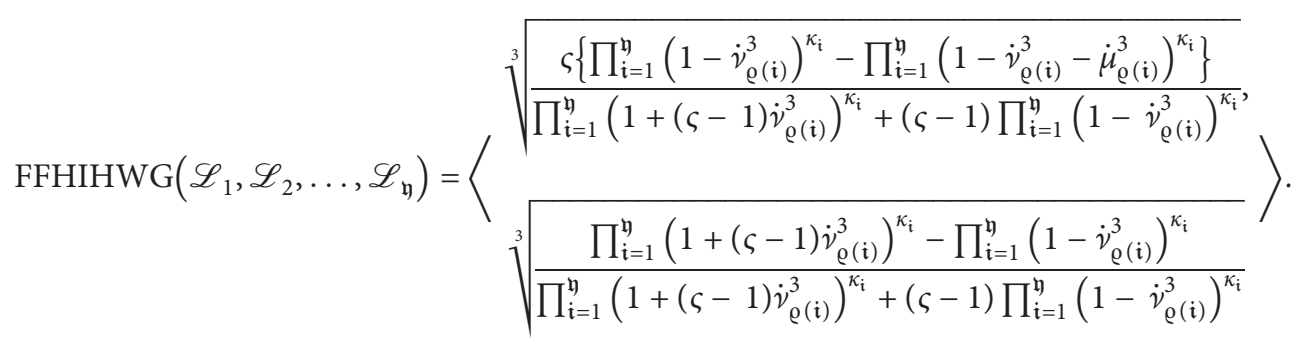

Proof. It is the same as that of Theorem 1.

Remark 3. The FFHIHWGO also satisfies the same results as given in Property 8.

\section{Decision-Making Analysis under FFE}

In a MADMM, it is a difficult task for decision makers to select the most feasible alternative among the given choices. Let $\left\{\mathfrak{S}_{1}, \mathfrak{S}_{2}, \ldots, \mathfrak{S}_{\mathfrak{y}}\right\}$ be $\mathfrak{y}$ distinct alternatives that can be characterized under a set of $m$ different criteria $\left\{c_{1}, c_{2}, \ldots, c_{m}\right\}$ taken by decision makers. Suppose that decision makers express their preference values in terms of FFSs $\alpha_{\mathfrak{i} j}=\left(\mu_{\mathfrak{i} j}, v_{\mathfrak{i} j}\right)(\mathfrak{i}=1,2, \ldots, \mathfrak{y} ; j=1,2, \ldots, m)$, where $\mu_{i j}$ and $\nu_{i j}$ are the BD and NBD of each alternative, respectively, corresponding to the given attribute such that $0 \leq \mu_{i j}^{3}+\nu_{i j}^{3} \leq 1$. There are different steps of MADM problem, given as follows:

Step 1. Decision criteria can be grouped into two opposite categories, usually called the "benefit" and the "cost" criteria. Benefit criteria may be called "reward" criteria and cost criteria "regret" or "loss" criteria. A benefit criterion means that the higher an alternative scores in terms of it, the better the alternative is. The opposite is considered true for the cost criteria. The normalized Fermatean fuzzy decision matrix (FFDMx) is obtained by interchanging the assessment values of cost attributes (CAs) with benefit attributes (BAs) [36]; that is,

$$
\mathscr{P}_{i j}= \begin{cases}\alpha_{i j}^{c}, & \text { for CAs, } \\ \alpha_{\mathfrak{i} j}, & \text { for BAs. }\end{cases}
$$

Step 2. By using the DMx given in Step 1, the overall aggregated value of alternatives $\mathfrak{S}_{\mathfrak{i}}$ under different choices of attributes $c_{j}$ is obtained by using any of FFHIWG, FFHIOWG, or FFHIHWG operators.

Step 3. The score function is used to determine the score values of the alternatives.

Step 4. The alternatives $\left\{\mathfrak{S}_{1}, \mathfrak{S}_{2}, \ldots, \mathfrak{S}_{\mathfrak{n}}\right\}$ are firstly arranged in descending order according to the score values and after it, the most appropriate alternative is selected.

A flowchart for the proposed MADM is shown in Figure 1.

6.1. Numerical Example. This section presents an application of the proposed MADM model in the field of medical science. Suppose that a doctor wants to select a patient who is more affected by cancer. Let $\left\{A_{1}, A_{2}, A_{3}, A_{4}, A_{5}\right\}$ be a set of patients and $\left\{C_{1}, C_{2}, C_{3}, C_{4}\right\}$ be a set of attributes with $\mathrm{WV}$ $\{0.3,0.3,0.2,0.2\}$. The following are the most important factors which increase the chances of cancer in a patient:

$$
\begin{aligned}
& C_{1} \text { stands for tobacco, } \\
& C_{2} \text { stands for alcohol, } \\
& C_{3} \text { stands for unhealthy diet, } \\
& C_{4} \text { stands for physical inactivity. }
\end{aligned}
$$

By FFHIWG operator, see the following

Step 1: since the criteria set is of the same type, DMx cannot be normalized.

Step 2: to determine the combined assessment of each alternative, we apply the FFHIWGO for $\varsigma=2$ as follows.

$$
\text { For } \mathscr{P}_{1} \text {, }
$$

$$
\begin{aligned}
& =\operatorname{FFHIWG}\left(\mathfrak{S}_{11}, \mathfrak{S}_{12}, \mathfrak{S}_{13}, \mathfrak{S}_{14}\right) \\
& =\left\langle\begin{array}{c}
\left.\frac{\sqrt{\frac{2\left(\left(1-0.6^{3}\right)^{0.3}\left(1-0.1^{3}\right)^{0.3}\left(1-0.4^{3}\right)^{0.2}\left(1-0.3^{3}\right)^{0.2}-\left(1-0.6^{3}-0.9^{3}\right)^{0.3}\left(1-0.1^{3}-0.6^{3}\right)^{0.3}\left(1-0.4^{3}-0.9^{3}\right)^{0.2}\left(1-0.3^{3}-0.5^{3}\right)^{0.2}\right)}{\left(1+0.6^{3}\right)^{0.3}\left(1+0.1^{3}\right)^{0.3}\left(1+0.4^{3}\right)^{0.2}\left(1+0.3^{3}\right)^{0.2}+\left(1-0.6^{3}\right)^{0.3}\left(1-0.1^{3}\right)^{0.3}\left(1-0.4^{3}\right)^{0.2}\left(1-0.3^{3}\right)^{0.2}}}}{\sqrt[3]{\frac{\left(1+0.6^{3}\right)^{0.3}\left(1+0.1^{3}\right)^{0.3}\left(1+0.4^{3}\right)^{0.2}\left(1+0.3^{3}\right)^{0.2}-\left(1-0.6^{3}\right)^{0.3}\left(1-0.1^{3}\right)^{0.3}\left(1-0.4^{3}\right)^{0.2}\left(1-0.3^{3}\right)^{0.2}}{\left(1+0.6^{3}\right)^{0.3}\left(1+0.1^{3}\right)^{0.3}\left(1+0.4^{3}\right)^{0.2}\left(1+0.3^{3}\right)^{0.2}+\left(1-0.6^{3}\right)^{0.3}\left(1-0.1^{3}\right)^{0.3}\left(1-0.4^{3}\right)^{0.2}\left(1-0.3^{3}\right)^{0.2}}}}\right\rangle
\end{array}\right.
\end{aligned}
$$

$=(0.87,0.56)$. 


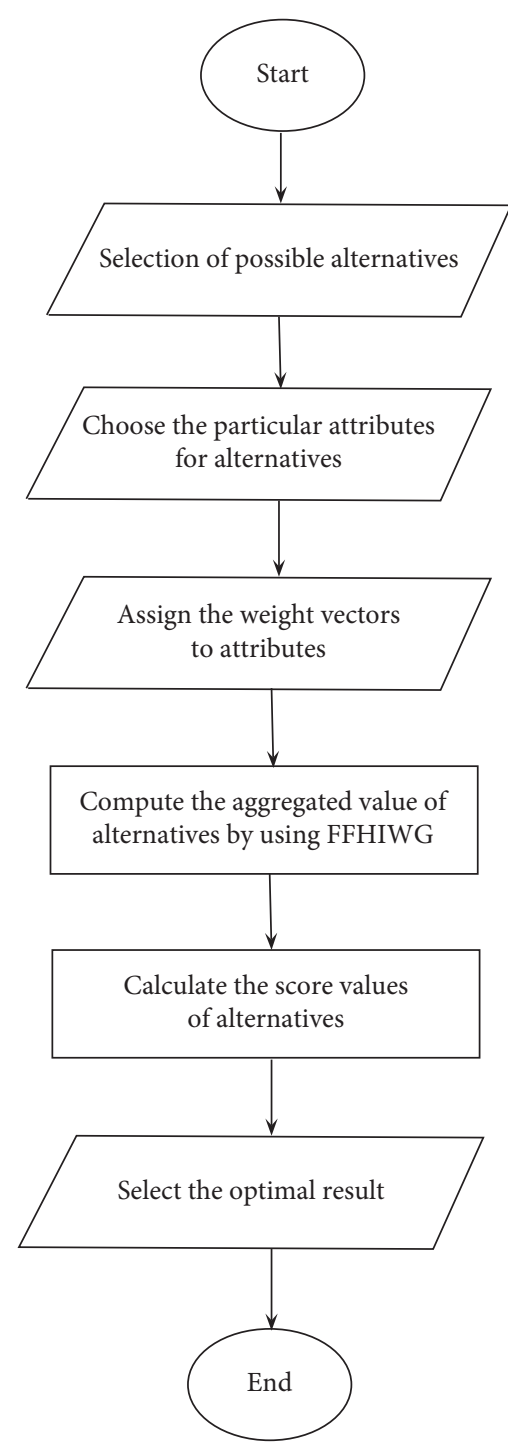

FIgURE 1: Flowchart for the selection of the best alternative.

For $\mathscr{P}_{2}$,

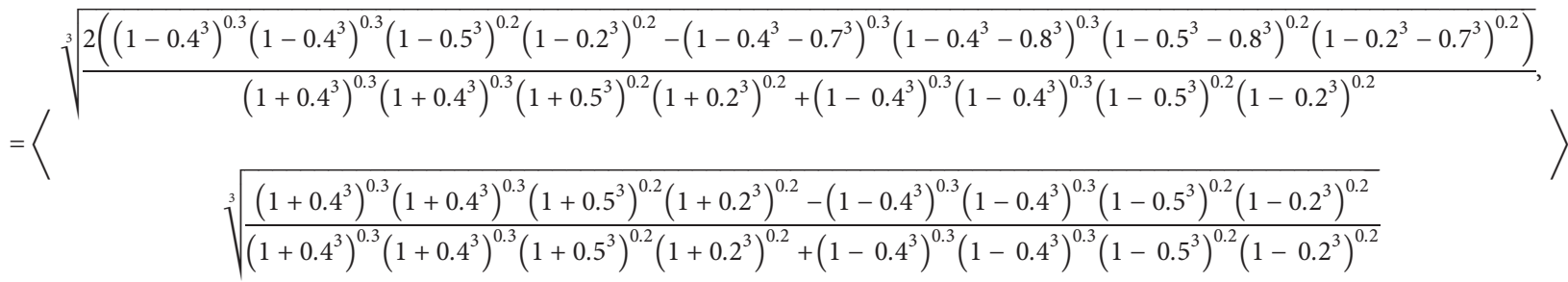

$=(0.76,0.37)$.

For $\mathscr{P}_{3}$, 
$=\left\langle\begin{array}{c}\left.\frac{\sqrt[3]{\frac{\left.2\left(1-0.6^{3}\right)^{0.3}\left(1-0.6^{3}\right)^{0.3}\left(1-0.6^{3}\right)^{0.2}\left(1-0.4^{3}\right)^{0.2}-\left(1-0.6^{3}-0.7^{3}\right)^{0.3}\left(1-0.6^{3}-0.4^{3}\right)^{0.3}\left(1-0.6^{3}-0.9^{3}\right)^{0.2}\left(1-0.4^{3}-0.8^{3}\right)^{0.2}\right)}{\left(1+0.6^{3}\right)^{0.3}\left(1+0.6^{3}\right)^{0.3}\left(1+0.6^{3}\right)^{0.2}\left(1+0.4^{3}\right)^{0.2}+\left(1-0.6^{3}\right)^{0.3}\left(1-0.6^{3}\right)^{0.3}\left(1-0.6^{3}\right)^{0.2}\left(1-0.4^{3}\right)^{0.2}}}}{\sqrt[3]{\frac{\left(1+0.6^{3}\right)^{0.3}\left(1+0.6^{3}\right)^{0.3}\left(1+0.6^{3}\right)^{0.2}\left(1+0.4^{3}\right)^{0.2}-\left(1-0.6^{3}\right)^{0.3}\left(1-0.6^{3}\right)^{0.3}\left(1-0.6^{3}\right)^{0.2}\left(1-0.4^{3}\right)^{0.2}}{\left(1+0.6^{3}\right)^{0.3}\left(1+0.6^{3}\right)^{0.3}\left(1+0.6^{3}\right)^{0.2}\left(1+0.4^{3}\right)^{0.2}+\left(1-0.6^{3}\right)^{0.3}\left(1-0.6^{3}\right)^{0.3}\left(1-0.6^{3}\right)^{0.2}\left(1-0.4^{3}\right)^{0.2}}}}\right\rangle\end{array}\right.$

$=(0.78,0.57)$.

For $\mathscr{P}_{4}$,

$=\operatorname{FFHIWG}\left(\mathfrak{S}_{11}, \mathfrak{S}_{12}, \mathfrak{S}_{13}, \mathfrak{S}_{14}\right)$

$=\left\langle\begin{array}{c}\left.\frac{\sqrt[3]{\frac{2\left(\left(1-0.2^{3}\right)^{0.3}\left(1-0.1^{3}\right)^{0.3}\left(1-0.4^{3}\right)^{0.2}\left(1-0.2^{3}\right)^{0.2}-\left(1-0.2^{3}-0.9^{3}\right)^{0.3}\left(1-0.1^{3}-0.9^{3}\right)^{0.3}\left(1-0.4^{3}-0.7^{3}\right)^{0.2}\left(1-0.2^{3}-0.4^{3}\right)^{0.2}\right)}{\left(1+0.2^{3}\right)^{0.3}\left(1+0.1^{3}\right)^{0.3}\left(1+0.4^{3}\right)^{0.2}\left(1+-0.2^{3}\right)^{0.2}+\left(1-0.2^{3}\right)^{0.3}\left(1-0.1^{3}\right)^{0.3}\left(1-0.4^{3}\right)^{0.2}\left(1-0.2^{3}\right)^{0.2}}}}{\sqrt[3]{\frac{\left(1+0.2^{3}\right)^{0.3}\left(1+0.1^{3}\right)^{0.3}\left(1+0.4^{3}\right)^{0.2}\left(1+-0.2^{3}\right)^{0.2}-\left(1-0.2^{3}\right)^{0.3}\left(1-0.1^{3}\right)^{0.3}\left(1-0.4^{3}\right)^{0.2}\left(1-0.2^{3}\right)^{0.2}}{\left(1+0.2^{3}\right)^{0.3}\left(1+0.1^{3}\right)^{0.3}\left(1+0.4^{3}\right)^{0.2}\left(1+-0.2^{3}\right)^{0.2}+\left(1-0.2^{3}\right)^{0.3}\left(1-0.1^{3}\right)^{0.3}\left(1-0.4^{3}\right)^{0.2}\left(1-0.2^{3}\right)^{0.2}}}}\right\rangle\end{array}\right.$

$=(0.92,0.27)$.

For $\mathscr{P}_{5}$,

$=\operatorname{FFHIWG}\left(\mathfrak{S}_{11}, \mathfrak{S}_{12}, \mathfrak{S}_{13}, \mathfrak{S}_{14}\right)$

$$
\begin{aligned}
& =\left\langle\sqrt[3]{\frac{2\left(\left(1-0.1^{3}\right)^{0.3}\left(1-0.4^{3}\right)^{0.3}\left(1-0.3^{3}\right)^{0.2}\left(1-0.4^{3}\right)^{0.2}-\left(1-0.1^{3}-0.8^{3}\right)^{0.3}\left(1-0.4^{3}-0.7^{3}\right)^{0.3}\left(1-0.3^{3}-0.6^{3}\right)^{0.2}\left(1-0.4^{3}-0.7^{3}\right)^{0.2}\right)}{\left(1+0.1^{3}\right)^{0.3}\left(1+0.4^{3}\right)^{0.3}\left(1+0.3^{3}\right)^{0.2}\left(1+0.4^{3}\right)^{0.2}+\left(1-0.1^{3}\right)^{0.3}\left(1-0.4^{3}\right)^{0.3}\left(1-0.3^{3}\right)^{0.2}\left(1-0.4^{3}\right)^{0.2}}},\right. \\
& \sqrt[3]{\frac{\left(1+0.1^{3}\right)^{0.3}\left(1+0.4^{3}\right)^{0.3}\left(1+0.3^{3}\right)^{0.2}\left(1+0.4^{3}\right)^{0.2}-\left(1-0.1^{3}\right)^{0.3}\left(1-0.4^{3}\right)^{0.3}\left(1-0.3^{3}\right)^{0.2}\left(1-0.4^{3}\right)^{0.2}}{\left(1+0.1^{3}\right)^{0.3}\left(1+0.4^{3}\right)^{0.3}\left(1+0.3^{3}\right)^{0.2}\left(1+0.4^{3}\right)^{0.2}+\left(1-0.1^{3}\right)^{0.3}\left(1-0.4^{3}\right)^{0.3}\left(1-0.3^{3}\right)^{0.2}\left(1-0.4^{3}\right)^{0.2}}}
\end{aligned}
$$

$=(0.72,0.34)$.

Step 3: the score values for each alternative are given as

$$
\begin{aligned}
& S\left(\widetilde{\Xi}_{1}\right)=0.56 \text {, } \\
& S\left(\widetilde{S}_{2}\right)=0.37 \text {, } \\
& S\left(\mathbb{S}_{3}\right)=0.29 \text {, } \\
& S\left(\mathfrak{S}_{4}\right)=0.76 \text {, } \\
& S\left(\mathfrak{S}_{5}\right)=0.33 \text {. }
\end{aligned}
$$

Step 4: as $\mathfrak{S}_{4}>\mathfrak{S}_{1}>\mathfrak{S}_{2}>\mathfrak{S}_{5}>\mathfrak{S}_{3}$, hence, $C_{4}$ is the person, who is most affected by cancer.

\section{Influence of Distinct Parameter's Values}

An attribute $\varsigma$ performs a crucial role in ranking results. In this section, we observe the score functions and ranking results based on FFHIWGO, under different values of $\varsigma$. Here, we observed some of the following influences:

(1) The influence of attributes $\varsigma$ on alternatives' ranking by applying the FFHIWGO

(2) The effect of attributes $\varsigma$ on alternatives' ranking by using the FFHIOWGO 
TABLE 1: Ranking order using the FFHIWG operator.

\begin{tabular}{ccccccc}
\hline$\varsigma$ & $S\left(A_{1}\right)$ & $S\left(A_{2}\right)$ & $S\left(A_{3}\right)$ & $S\left(A_{4}\right)$ & $S\left(A_{5}\right)$ & Ranking order \\
\hline 1 & 0.54 & 0.37 & 0.32 & 0.76 & 0.33 & $A_{4}>A_{1}>A_{2}>A_{5}>A_{3}$ \\
2 & 0.56 & 0.37 & 0.29 & 0.76 & 0.33 & $A_{4}>A_{1}>A_{2}>A_{5}>A_{3}$ \\
3 & 0.56 & 0.37 & 0.29 & 0.76 & 0.32 & $A_{4}>A_{1}>A_{2}>A_{5}>A_{3}$ \\
4 & 0.58 & 0.37 & 0.29 & 0.76 & 0.34 & $A_{4}>A_{1}>A_{2}>A_{5}>A_{3}$ \\
5 & 0.58 & 0.37 & 0.31 & 0.76 & 0.33 & $A_{4}>A_{1}>A_{2}>A_{5}>A_{3}$ \\
\hline
\end{tabular}

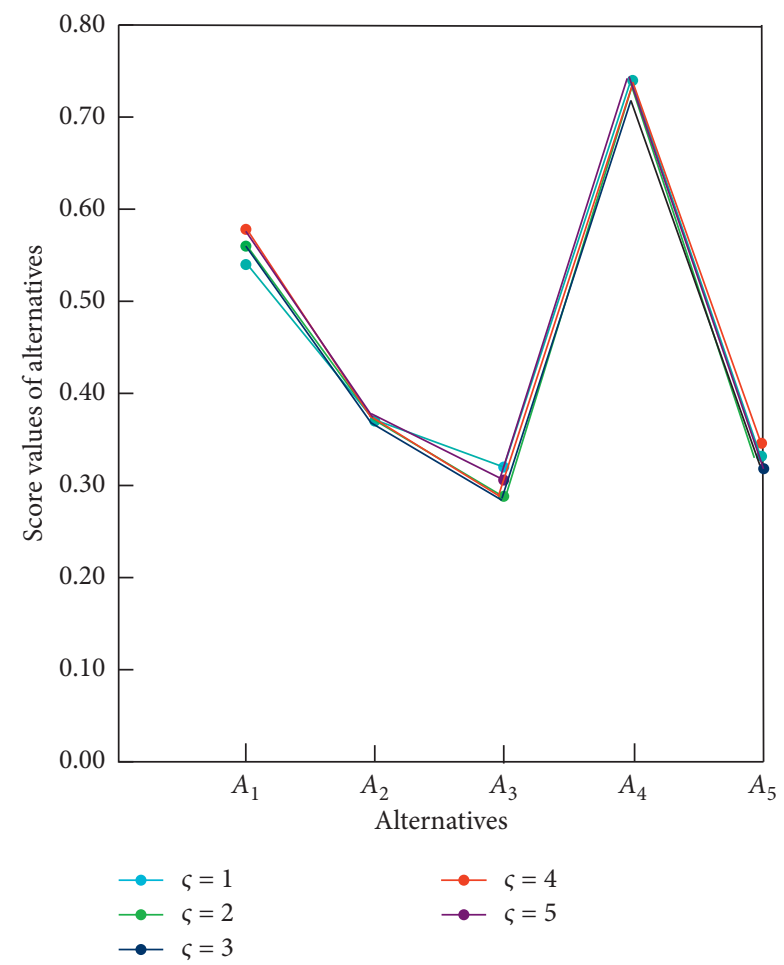

Figure 2: Ranking order for different values of $\varsigma$.

(3) The influence of attributes $\varsigma$ on alternatives' ranking by applying FFHIHWGO

From Table 1 and Figure 2, we can see that alternatives may have the same or different score values but the ranking order remains the same for all the attributes.

\section{A Comparative Analysis and Discussion}

To view the importance and validity of proposed AOs, we give a comparative analysis of the proposed model with the different existing models. From Table 2, it is clear that there are some values for which $\mu+\nu>1$ and $\mu^{2}+\nu^{2}>1$. Therefore, the methods presented in $[18,23]$ failed to handle such problems.

8.1. Comparison with Fermatean Fuzzy TOPSIS Method. This section presents the comparison of the proposed work with the FF-TOPSIS method [7]. The steps are given as follows:

(1) Table 2 provides the FFDM in which each entry is a FFS.
(2) The Fermatean fuzzy positive ideal solution (FFPIS) $\mathscr{H}^{+}$and Fermatean fuzzy negative ideal solution (FFNIS) $\mathscr{H}^{-}$are

$$
\begin{aligned}
& \mathscr{H}^{+}=\{(0.9,0.2),(0.9,0.1),(0.9,0.4),(0.8,0.4)\}, \\
& \mathscr{H}^{-}=\{(0.7,0.6),(0.4,0.6),(0.6,0.3),(0.4,0.2)\} .
\end{aligned}
$$

(3) The distance of alternatives $A_{i}$ from FFPIS $\mathscr{H}^{+}$and FFNIS $\mathscr{H}^{-}$is given in Table 3.

(4) The revised closeness degree of each alternative is given:

$$
\begin{aligned}
& \xi\left(A_{1}\right)=-0.92, \\
& \xi\left(A_{2}\right)=-0.45, \\
& \xi\left(A_{3}\right)=-1.038, \\
& \xi\left(A_{4}\right)=0, \\
& \xi\left(A_{5}\right)=-1.16 .
\end{aligned}
$$

(5) Arrange the alternatives in descending order with respect to $\xi\left(A_{i}\right)$ : 
TABLE 2: FF decision matrix.

\begin{tabular}{ccccc}
\hline$\alpha_{i j}$ & $C_{1}$ & $C_{2}$ & $C_{3}$ & $C_{4}$ \\
\hline$A_{1}$ & $(0.9,0.6)$ & $(0.6,0.1)$ & $(0.9,0.4)$ & $(0.5,0.3)$ \\
$A_{2}$ & $(0.7,0.4)$ & $(0.8,0.4)$ & $(0.8,0.5)$ & $(0.7,0.2)$ \\
$A_{3}$ & $(0.7,0.6)$ & $(0.4,0.6)$ & $(0.9,0.6)$ & $(0.8,0.4)$ \\
$A_{4}$ & $(0.9,0.2)$ & $(0.9,0.1)$ & $(0.7,0.4)$ & $(0.4,0.2)$ \\
$A_{5}$ & $(0.8,0.1)$ & $(0.7,0.4)$ & $(0.6,0.3)$ & $(0.7,0.4)$ \\
\hline
\end{tabular}

TABLE 3: Distance of alternatives from FFPIS and FFNIS.

\begin{tabular}{lc}
\hline$D\left(A_{i}, \mathscr{H}^{+}\right)$ & $D\left(A_{i}, \mathscr{H}^{-}\right)$ \\
\hline 0.298 & 0.242 \\
0.245 & 0.293 \\
0.307 & 0.222 \\
0.174 & 0.306 \\
0.314 & 0.196 \\
\hline
\end{tabular}

$$
A_{4}>A_{2}>A_{1}>A_{3}>A_{5} .
$$

(6) Thus, $A_{4}$ is the best alternative.

It is clear that ranking lists is slightly different from both methods but the most suitable alternative from both methods is $A_{4}$. Though, FF-TOPSIS method is a useful technique to solve MADMMs, but there are also some issues which cannot be resolved by adopting this method. Therefore, such issues can be tackled through the proposed model and the results obtained from it are closer to the original results.

Advantages and limitations of intended AOs: the benefit and motivation behind this intended approach are as follows:

(i) The other membership grades in the aggregated value affect the other grades even if $\mathrm{BD}$ of any alternative is zero.

(ii) There is a relationship between the BD and NBD of an alternative.

(iii) However, there are some limitations of this model. It cannot be applied in situations where we take the parameters for the evaluation of anything. It means this theory has a lack of parametrization property.

\section{Conclusions}

A FFS having emerging applications in MADM, is a more efficient technique to cope with ambiguities involved in the given data, as compared to IFSs and PFSs. AOs are very useful for evaluating the given alternatives in DM process because they integrate the evaluation values of all the given individuals into a unified form. By comparing other AOs, the structure of Hamacher's norms is a more general framework, which effectively integrates complex information. In this article, we have intended some FF-Hamacher interactive geometric AOs such as FFHIWGO, FFHIOWGO, and FFHIHWGO. These intended operators have some useful characteristics, for example, boundedness, homogeneity, idempotency, monotonicity, and shift invariance. Some special cases of the proposed AOs have been also explained. The interaction between belongingness and nonbelongingness degrees has been discussed in the proposed HIAOs. We have explained a MADM algorithm to cope with uncertainties present in decision problems. To show the efficacy and applicability of the intended MADM, we have applied this method in a real-life system. We have presented a comparative analysis of our model with some of the existing models and concluded that our model is more flexible and it depicts ambiguous and inexact information in complex structures. Thus, the AOs due to their highly adaptable nature are very crucial. Therefore, taking into account this direction further we will explore some more properties and types of these operators in Fermatean fuzzy soft sets and $q$-rung orthopair fuzzy soft sets. Moreover, we will extend our work on the following decision-making problems:

(1) An in-depth study of the Hamacher AOs for Fermatean fuzzy information such as induced Fermatean fuzzy Hamacher interactive AOs and q-rung picture fuzzy Hamacher interactive AOs will be a hot topic in the future

(2) A MADM problem in medical diagnosis under Fermatean fuzzy soft data using Hamacher interactive AOs will be discussed

(3) A MADM problem for the selection of a smartphone under Fermatean fuzzy soft data using Hamacher interactive AOs will be discussed

\section{Data Availability}

No data were used to support this study.

\section{Ethical Approval}

This article does not contain any studies with human participants or animals performed by any of the authors.

\section{Conflicts of Interest}

The authors declare no conflicts of interest.

\section{Acknowledgments}

This project was supported by Imam Abdulrahman Bin Faisal University, Dammam, Saudi Arabia. 


\section{References}

[1] L. A. Zadeh, "Fuzzy sets," Information and Control, vol. 8, no. 3, pp. 338-353, 1965.

[2] K. T. Atanassov, "Intuitionistic fuzzy sets," Fuzzy Sets and Systems, vol. 20, no. 1, pp. 87-96, 1986.

[3] R. R. Yager, "Pythagorean fuzzy subsets," in Proceedings of the 2013 Joint IFSA World Congress and NAFIPS Annual Meeting (IFSA/NAFIPS), pp. 57-61, IEEE, Edmonton, Canada, June 2013.

[4] V. Torra, "Hesitant fuzzy sets," International Journal of Intelligent Systems, vol. 25, no. 6, pp. 529-539, 2010.

[5] V. Torra and Y. Narukawa, "On hesitant fuzzy sets and decision," in Proceedings of the IEEE International Conference on Fuzzy Systems, pp. 1378-1382, Jeju-do, Korea, August 2009.

[6] L. A. Zadeh, "The concept of a lingusistic variable and its application to approximate reasoning," Information Sciences, vol. 8, no. 3, pp. 199-249, 1975.

[7] T. Senapati and R. R. Yager, "Fermatean fuzzy sets," Journal of Ambient Intelligence and Humanized Computing, vol. 11, no. 2, pp. 663-674, 2020.

[8] Z. Xu, "Intuitionistic fuzzy aggregation operators," IEEE Transactions on Fuzzy Systems, vol. 15, no. 6, pp. 1179-1187, 2007.

[9] H. Zhao, Z. Xu, M. Ni, and S. Liu, "Generalized aggregation operators for intuitionistic fuzzy sets," International Journal of Intelligent Systems, vol. 25, no. 1, pp. 1-30, 2010.

[10] G. Wei and M. Lu, "Pythagorean fuzzy power aggregation operators in multiple attribute decision making," International Journal of Intelligent Systems, vol. 33, no. 1, pp. 169-186, 2018.

[11] R. R. Yager, "On ordered weighted averaging aggregation operators in multicriteria decisionmaking," IEEE Transactions on Systems, Man, and Cybernetics, vol. 18, no. 1, pp. 183-190, 1988.

[12] J. Dombi, “A general class of fuzzy operators, the demorgan class of fuzzy operators and fuzziness measures induced by fuzzy operators," Fuzzy Sets and Systems, vol. 8, no. 2, pp. 149-163, 1982.

[13] M. Akram, W. A. Dudek, and J. M. Dar, "Pythagorean dombi fuzzy aggregation operators with application in multi-criteria decision making," International Journal of Intelligent Systems, vol. 34, no. 11, pp. 3000-3019, 2019.

[14] G. Wei, "Pythagorean fuzzy interaction aggregation operators and their application to multiple attribute decision making," Journal of Intelligent and Fuzzy Systems, vol. 33, no. 4, pp. 2119-2132, 2017.

[15] H. Hamacher, "Uber Logische Verknunpfungenn Unssharfer Aussagen und deren Zugenhorige Bewertungsfunktione," in Progress in Cybernatics and Systems Research, R. Trappl, G. H. Klir, and L. Riccardi, Eds., vol. 3, pp. 276-288, Hemisphere, Washington, DC, 1978.

[16] G. Wei, "Pythagorean fuzzy hamacher power aggregation operators in multiple attribute decision making," Fundamenta Informaticae, vol. 166, no. 1, pp. 57-85, 2019.

[17] H. Garg, "Some series of intuitionistic fuzzy interactive averaging aggregation operators," SpringerPlus, vol. 5, no. 1, p. 999, 2016.

[18] H. Garg, "Intuitionistic fuzzy hamacher aggregation operators with entropy weight and their applications to multi-criteria decision-making problems," Iranian Journal of Science and Technology, Transactions of Electrical Engineering, vol. 43, no. 3, pp. 597-613, 2019.
[19] S. J. Wu and G. W. Wei, "Pythagorean fuzzy hamacher aggregation operators and their application to multiple attribute decision making," International Journal of Knowledge-Based and Intelligent Engineering Systems, vol. 21, no. 3, pp. 189-201, 2017.

[20] N. Waseem, M. Akram, and J. C. R. Alcantud, "Multi-attribute decision making based on m-polar fuzzy hamacher aggregation operators," Symmetry, vol. 11, no. 12, p. 1498, 2019.

[21] X. Zhao and G. Wei, "Some intuitionistic fuzzy einstein hybrid aggregation operators and their application to multiple attribute decision making," Knowledge-Based Systems, vol. 37, pp. 472-479, 2013.

[22] W. Wang and X. Liu, "Intuitionistic fuzzy information aggregation using einstein operations," IEEE Transactions on Fuzzy Systems, vol. 20, no. 5, pp. 923-938, 2012.

[23] L. Wang, H. Garg, and N. Li, "Pythagorean fuzzy interactive hamacher power aggregation operators for assessment of express service quality with entropy weight," Soft Computing, vol. 25, no. 2, pp. 973-993, 2021.

[24] T. Senapati and R. R. Yager, "Fermatean fuzzy weighted averaging/geometric operators and its application in multicriteria decision making methods," Engineering Applications of Artificial Intelligence, vol. 85, pp. 112-121, 2019.

[25] T. Senapati and R. R. Yager, "Some new operations over fermatean fuzzy numbers and application of fermatean fuzzy WPM in multiple criteria decision making," Informatica, vol. 30, no. 2, pp. 391-412, 2019.

[26] H. Garg, G. Shahzadi, and M. Akram, "Decision-making analysis based on Fermatean fuzzy Yager aggregation operators with application in COVID-19 testing facility," Mathematical Problems in Engineering, vol. 2020, Article ID 7279027, 16 pages, 2020.

[27] M. Akram, G. Shahzadi, and A. A. H. Ahmadini, "Decision making framework for an effective sanitizer to reduce COVID-19 under fermatean fuzzy environment," Journal of Mathematics, vol. 2020, Article ID 3263407, 19 pages, 2020.

[28] G. Shahzadi and M. Akram, "Group decision-making for the selection of an antivirus mask under fermatean fuzzy soft information," Journal of Intelligent and Fuzzy Systems, vol. 40, no. 1, pp. 1401-1416, 2021.

[29] S. B. Aydemir and S. Y. Gunduz, "Fermatean fuzzy TOPSIS method with dombi aggregation operators and its application in multi-criteria decision making," Journal of Intelligent and Fuzzy Systems, vol. 39, no. 1, pp. 1-19, 2020.

[30] G. Shahzadi, G. Muhiuddin, M. Arif Butt, and A. Ashraf, "Hamacher interactive hybrid weighted averaging operators under fermatean fuzzy numbers," Journal of Mathematics, vol. 2020, Article ID 5556017, 17 pages, 2021.

[31] F. Feng, Y. Zheng, B. Sun, and M. Akram, "Novel score functions of generalized orthopair fuzzy membership grades with application to multiple attribute decision making," Granular Computing, 2021.

[32] M. Akram and G. Shahzadi, "A hybrid decision making model under q-rung orthopair fuzzy yager aggregation operators," Granular Computing, pp. 1-15, 2020.

[33] M. Akram, G. Shahzadi, M. A. Butt, and F. Karaaslan, "A hybrid decision making method based on q-rung orthopair fuzzy soft information," Journal of Intelligent and Fuzzy Systems, vol. 40, no. 5, pp. 9815-9830, 2021.

[34] M. Akram, G. Shahzadi, and X. Peng, "Extension of einstein geometric operators to multi-attribute decision making under q-rung orthopair fuzzy information," Granular Computing, pp. 1-17, 2020. 
[35] M. Akram, G. Shahzadi, and S. Shahzadi, "Protraction of einstein operators for decision-making under q-rung orthopair fuzzy model," Journal of Intelligent and Fuzzy Systems, vol. 40, no. 3, pp. 4779-4798, 2021.

[36] Z. Xu and H. Hu, "Projection models for intuitionistic fuzzy multiple attribute decision making," International Journal of Information Technology and Decision Making, vol. 9, no. 2, pp. 267-280, 2010. 